\title{
EFFECT OF DIFFERENT CHEMICAL RIPENERS ON SUGARCANE (SACCHARUM OFFICINARUM L.) QUALITY, SUGAR YIELD AND RATOONING ABILITIES
}

\author{
TUfAIL, M. - HusSAIN, K. ${ }^{*}$ \\ Department of Botany, University of Gujrat, Gujrat, Pakistan \\ *Corresponding author \\ e-mail: khalid.hussain@uog.edu.pk \\ (Received $3^{\text {rd }}$ Apr 2020; accepted $29^{\text {th }}$ Jul 2020)
}

\begin{abstract}
Field experiments were conducted to evaluate the impact of various chemical ripeners (Ethephon, Glyphosate, Sulfometuron-methyl) on sugarcane cultivars (HSF-242, NSG-311, HSF-240, NSG-555). Each chemical ripener (200 ppm) was applied as a foliar spray 15, 30 and 45 days before cane harvesting for planted crops and ratoons were collected. Chemical ripeners significantly enhanced the quality and sugar yielding traits including harvest index, juice extraction and purity, sugar recovery $\%$ cane and total sugar yield for planted crop especially when treatment was applied 45 days before cane harvesting. Changes in antioxidant activities indicated the influence of various chemical ripeners on the planted crop. Chemical ripeners were imperative to create short-term changes for early cane maturity and sugar yielding abilities. Performance of ratooning crop was normal concerning growth, yield and antioxidant activities according to its existing genetic makeup showing non-significant effect of chemical ripeners. It was concluded that chemical ripeners can be suitable to boost up sugar yielding characteristics by inducing early cane maturity for a short duration before cane harvesting and it will have no effect on lateral life cycle span and its ratooning abilities.
\end{abstract}

Keywords: glyphosate, ethephon, sulfometuron-methyl, sugar production

\section{Introduction}

Sugarcane (Saccharum officinarum L.) is considered as an industrial crop for the production of sugar (Neliana et al., 2019). Sugarcane is one of the world's major foodproducing crops, providing about $75 \%$ of sugar in the world for human consumption (De Souza, 2008). Sugarcane is rich in sucrose which is accumulated in stalk internodes and is used to manufacture many industrial goods such as furfural, alcohol, dextrans etc. and some other natural pharmaceutical products (Ma et al., 2005).

Chemical ripeners (Ethephon, ethyl-trinexapac, glyphosate and sulfometurom methyl) are classified as growth retardants and growth inhibitors as described by Leite et al. (2011). Chemical ripening of sugarcane is an important component to profitable sugar production throughout the world. Harvesting of sugarcane often begins before the sugarcane reaches the desirable maturity level (Dalley and Richard-Junior, 2010). The main advantage of chemical ripeners is that they can suppress stalk and leaf growth much more rapidly and consistently than natural processes such as reduced temperatures or limiting soil moisture (Van Heerden et al., 2015). Ethephon, an ethylene releasing compound, was the first growth regulator in the early 1960s used for crop management and post-harvest quality in a wide range of agricultural, horticultural and forestry. The application of Ethephon in sugarcane has accelerated ripening, increased the overall sugar yield, and inhibited flowering (De Almeida and Caputo, 2012).

Glyphosate, an amino acid synthesis inhibitor, applied at sub-lethal doses has been widely used to increase sucrose levels in sugarcane (Solomon and Li, 2004). Glyphosate 
(N-phosphonomethyl glycine, $\mathrm{C}_{3} \mathrm{H}_{8} \mathrm{NO}_{5} \mathrm{P}$ ) is the analogue of glycine. It is a highly used herbicide throughout the world because it is an efficient killer of weeds, less toxic and available at low cost (Goscinny and Hanot, 2012). Leite et al. (2009) noted that ripener application for early harvest sugarcane led to an increase in technological quality, although sugar yield had been significantly affected, which positively contributed to the profit per unit area. El-Hamd et al. (2013) found that glyphosate application increased total soluble solids in cane juice but other quality parameters viz. sucrose content also increased proportionately. The introduction of sulfometuron-methyl, which overcomes some of the disadvantages of other ripeners, is therefore timely. Sulfometuron-methyl is a grass herbicide that showed promise as a chemical at low rates of application (Almendares et al., 2013). Many studies reported that sulfometuron-methyl regarding its potential ripening effect in sugarcane varieties, causes no damage to sugarcane production $\left(\mathrm{t} \mathrm{ha}^{-1}\right)$ or the agronomic characteristics of the culture (Silva et al., 2007; Leite et al., 2010).

There is a lack of information about the use of chemical ripeners in Pakistan for sugarcane. There are different studies in few countries about the effective use of chemical ripeners for early cane ripening with higher sugar yield but there is no information and use of chemical ripeners in Pakistan. Secondly, residual effects of chemical ripeners has not been evaluated on sugarcane ratooning abilities. This study was conducted first time in Pakistan to find the efficacy of chemical ripeners on sugar yielding attribute on planted crop as well as its impact on the ratooning abilities.

\section{Materials and methods}

Experiments were carried out at Shakarganj Sugar Research Institute (SSRI) Jhang and University of Gujrat, Pakistan during 2018-19. Sowing of four sugarcane cultivars i.e. HSF-242, NSG-311, HSF-240 and NSG-555 was done in two sowing seasons i.e. spring and autumn during 2018 and 2019. Experimental design was RCBD (Plot size $30 \times 30$ feet beds) with four replicates. A seed rate of 75000 double-bedded setts per hectares was used with 2.5 feet row spacing. Seed was treated with hot water at $52{ }^{\circ} \mathrm{C}$ for $30 \mathrm{~min}$ and fungicide for better germination and to control sugarcane diseases. Soil insecticide was also applied in the soil to control termites. Double-cut setts were placed end to end in furrows at a depth of about $10 \mathrm{~cm}$ and covered with $5 \mathrm{~cm}$ soil. Immediate irrigation was applied after planting. Each chemical ripener was sprayed at cane formation and elongation phase. These concentrations have not been studied for sugarcane crop in previous research and with the treatment intervals of 15, 30 and 45 before cane harvesting. Researcher used less or more than $200 \mathrm{ppm}$ concentrations of different chemical ripeners in sugarcane and used single dose application that is why this interval of treatments was applied.

The following treatments were applied on planted crop:

$\mathrm{T} 0=$ Control

$\mathrm{T} 1=200$ ppm Ethephon spray (15 days before harvesting)

$\mathrm{T} 2=200 \mathrm{ppm}$ Ethephon spray (30 days before harvesting)

$\mathrm{T} 3=200$ ppmEthephon spray (45 days before harvesting)

$\mathrm{T} 4=200$ ppm Glyphosate spray (15 days before harvesting)

T5 $=200$ ppm Glyphosate spray (30 days before harvesting)

T6 $=200$ ppm Glyphosate spray (45 days before harvesting)

$\mathrm{T} 7=200 \mathrm{ppm}$ Sulfometuron-methyl spray (15 days before harvesting) 
T8 $=200$ ppm Sulfometuron-methyl spray (30 days before harvesting)

T9 $=200$ ppm Sulfometuron-methyl spray (45 days before harvesting)

The following quality, sugar yielding and antioxidant activities were determined for planted crop in January-February, 2019:

1. Harvest Index (\%)

2. Juice Extraction (\%)

3. Juice purity $(\%)$

4. Sugar recovery $\%$ cane

5. Sugar yield $\left(\mathrm{t} \mathrm{ha}^{-1}\right)$

6. Antioxidant activities (CAT, POD and SOD)

After the cane harvesting, ratoon was kept from the planted crop at which chemical ripeners were sprayed and the following parameters were studied in January-February, 2020:

1. Ratoon Sprouting (\%)

2. Number of tillers per plant

3. Mill-able canes $\left(\mathrm{t} \mathrm{ha}^{-1}\right)$

4. Juice Extraction (\%)

5. Juice purity $(\%)$

6. Sugar recovery \% cane

7. Sugar yield $\left(\mathrm{t} \mathrm{ha}^{-1}\right)$

8. Total cane yield $\left(\mathrm{t} \mathrm{ha}^{-1}\right)$

9. Antioxidant activities (CAT, POD and SOD)

Harvest index (HI) was calculated for planted crop using this formula:

$$
\mathrm{HI}(\%)=\text { Stripped cane yield/Unstripped cane yield } \mathrm{x} 100
$$

Juice extraction \% was calculated both for planted and ratooning crop by this formula:

$$
\text { Juice extraction }(\%)=\text { Juice weight }(\mathrm{g}) / \text { Filter cake weight }(\mathrm{g}) \times 100
$$

Sugar recovery $\%$ of cane for planted and ratooning crop was calculated by using the formula as follows:

$$
\text { Sugar recovery percentage }=[S-0.4(B-S)] \times 0.73
$$

where: $\mathrm{B}=$ Brix percentage, $\mathrm{S}=$ sucrose percentage, 0.4 and 0.73 constant factors.

Total sugar yield $\left(\mathrm{t} \mathrm{ha}^{-1}\right)$ was calculated for each treatment by the following method:

$$
\text { Total sugar }\left(\mathrm{t} \mathrm{ha}^{-1}\right)=\text { Sugar recovery } \mathrm{x} \text { Stripped-cane yield } / 100
$$

Estimation of CAT, POD and SOD activities were determined by the procedure of Chance and Maehly (1955).

Data were analyzed statistically using analysis of variance (ANOVA) technique using Ministate-C software and significant mean separation was done at $\mathrm{P} \leq 0.05$ using Tukey's test. 


\section{Results}

The following results were obtained by the applications of chemical ripeners in planted and ratooning crop.

\section{Harvest index (HI) \% for planted crop}

Effect of chemical ripeners was highly significant in sugarcane for harvest index (Table 1). There were significant variations among sugarcane cultivars for $\mathrm{HI}$ and its interaction between ripeners and cultivars. Higher HI value was noted in cultivar HSF240 with the treatment of Ethephon and Sulfometuron-methyl that was applied 45 days before cane harvesting. Cultivar NSG-555 showed the lowest HI values for all the treatments of ripeners (Fig. 1A). Overall, all the treatments that were applied 45 days before harvesting had the highest values for HI as compared to other treatments (Table 2). HI increased because chemical ripeners helped to produce maximum number of stripped cane.

\section{Juice purity (\%) in planted crop}

It was noted from the results that the effect of chemical ripeners was highly significant for juice purity $\%$ of sugarcane. Variations among cultivars were significant while the interaction between ripeners x cultivar was highly significant (Table 1). Higher juice purity \% was calculated in NSG-555 and the lowest juice purity was present in HSF-242 (Table 2). Applications of 200 ppm of Glyphosate and Ethephon that were applied 45 days before harvesting showed better results for juice purity (\%). All the chemical ripeners applied 45 days before harvesting increased juice purity $\%$ (Fig. 1C).

\section{Sugar recovery \% of cane in planted crop}

Sugar recovery $\%$ of cane significantly increased with the applications of chemical ripeners. There were highly significant results for sugar recovery $\%$ of cane among cultivars as well as in interactions of ripeners x cultivar (Table 1). The changes in sugar recovery $\%$ of cane was due to the changes created by chemical ripeners in juice extraction and purity \%. Higher sugar recovery \% of cane was obtained in NSG-555 by the applications of $200 \mathrm{ppm}$ Glyphosate that were applied 45 days before cane harvesting. HSF-242 produced the lowest sugar recovery \% of cane (Fig. 2A). All the chemical ripeners increased the sugar recovery $\%$ of cane but the treatments applied before 45 days were the best sugar recovery $\%$ of cane (Table 2).

\section{Juice extraction (\%) from planted crop}

Data presented for juice extraction showed that chemical ripeners had highly significant juice extraction \% (Table 1). Interaction of ripeners $\mathrm{x}$ cultivar were also highly significant. Cultivars had also highly significant response to chemical ripeners. Juice extraction \% was increased by the treatments of chemical ripeners (Fig. 1B). Higher juice was extracted from HSF-240 with the applications of 200 ppm Ethephon that was applied 45 days before harvesting. Overall, NSG-555 cultivar had the highest juice extraction while the lowest juice quantity was extracted from HSF-242. From the results it was apparent that chemical ripeners produced early maturity and increased the production of juice in sugarcane (Table 2). All the chemicals applied 45 days before 
harvesting were the best in juice extraction as compared to the treatments sprayed 15 and 30 days before cane harvesting.

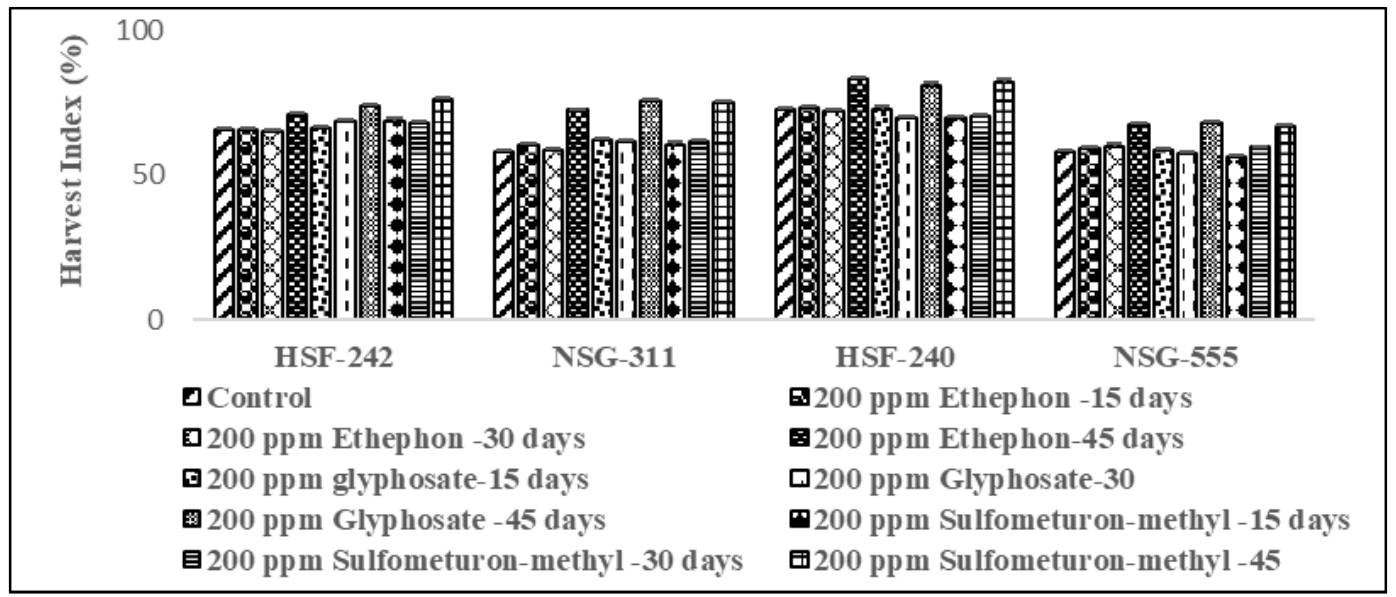

A: Harvest index

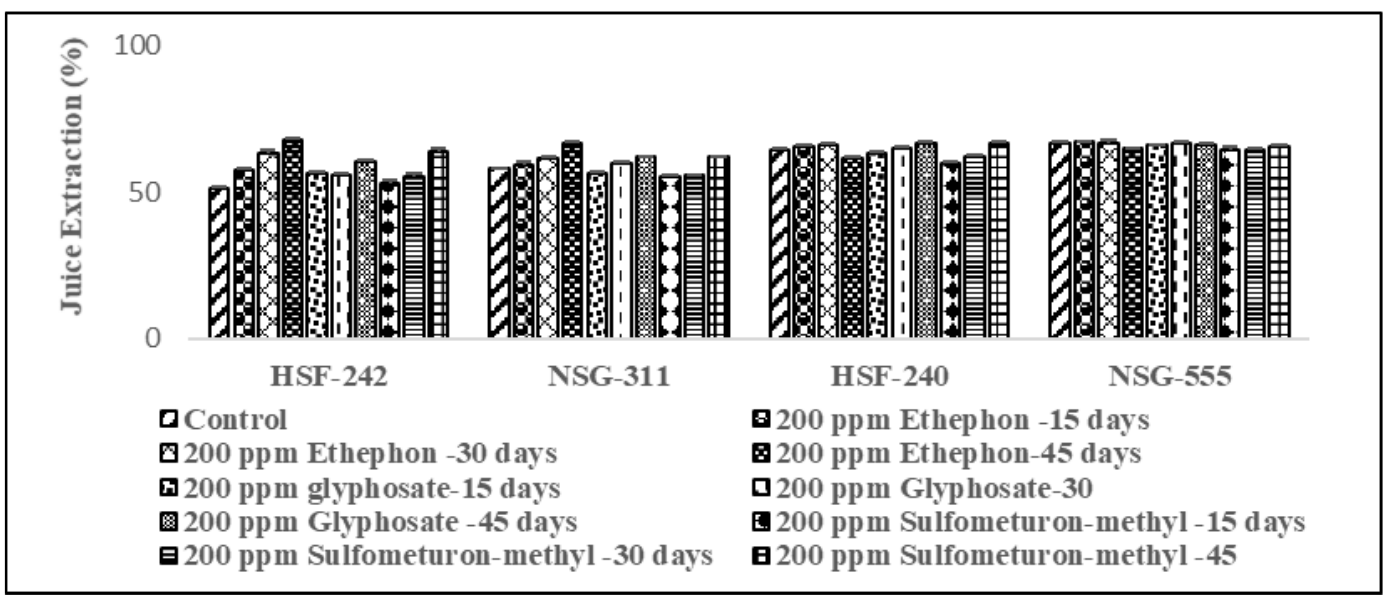

B: Juice extraction

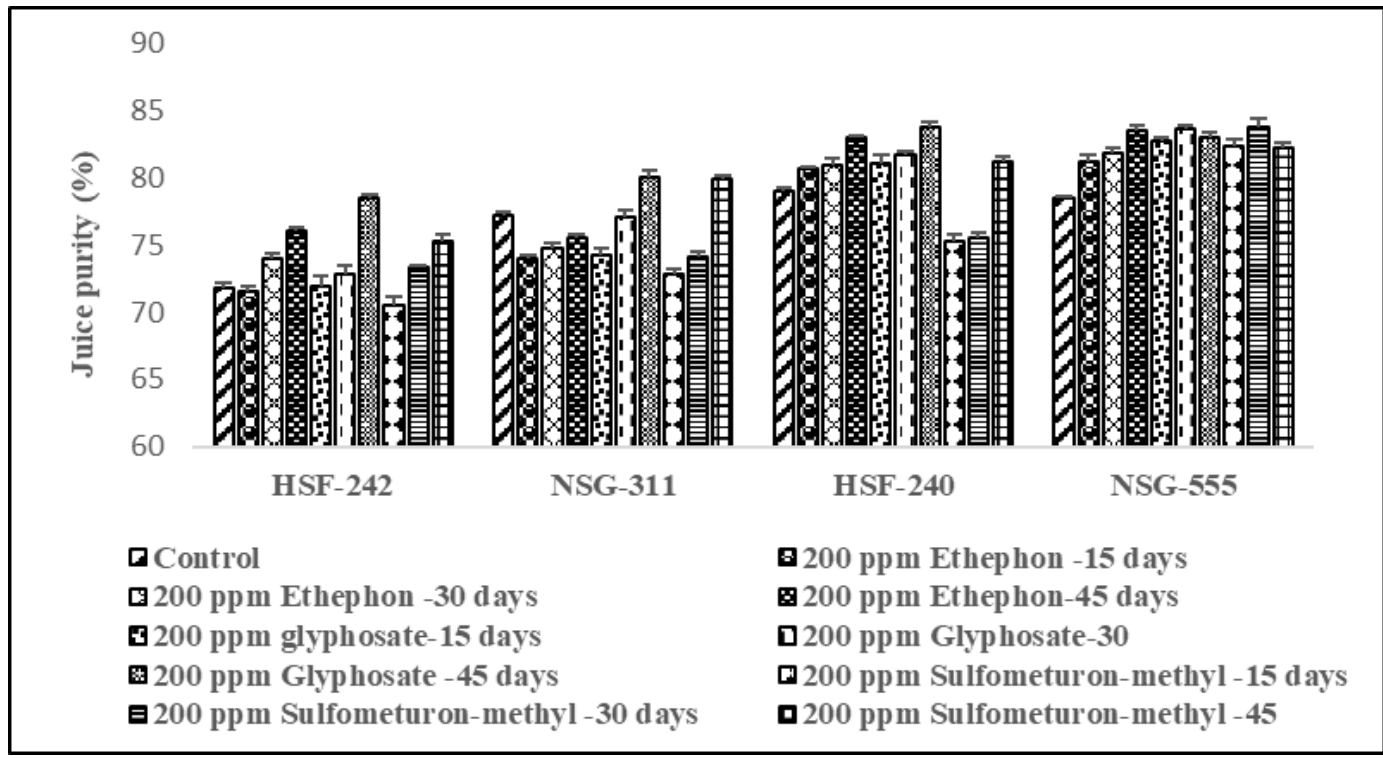

C: Juice purity

Figure 1. Effect of different chemical ripeners on quality attributes of sugarcane cultivars in planted crop 
Table 1. Mean squares (MS) from the analysis of variance (ANOVA) for quality and antioxidant activities of planted sugarcane cultivars under the applications of chemical ripeners

\begin{tabular}{|c|c|c|c|c|c|c|c|c|c|}
\hline Source & df & $\begin{array}{l}\text { Harvest } \\
\text { index }(\%)\end{array}$ & $\begin{array}{c}\text { Juice } \\
\text { extraction } \\
(\%)\end{array}$ & $\begin{array}{c}\text { Juice purity } \\
(\%)\end{array}$ & $\begin{array}{c}\text { Sugar } \\
\text { recovery \% } \\
\text { cane }\end{array}$ & $\begin{array}{l}\text { Total sugar } \\
\text { yield }\left(\mathbf{t h a}^{-1}\right)\end{array}$ & $\begin{array}{l}\text { Peroxidase } \\
\text { (POD) } \\
\text { activities }\end{array}$ & $\begin{array}{c}\text { Catalases } \\
(\text { CAT) } \\
\text { activities }\end{array}$ & $\begin{array}{c}\text { Superoxide } \\
\text { dismutase } \\
\text { (SOD) } \\
\text { activities }\end{array}$ \\
\hline $\begin{array}{l}\text { Main effects } \\
\text { Ripeners } \\
\text { (Rip) }\end{array}$ & 9 & $62.579 * * *$ & $97.439 * * *$ & $52.524 * * *$ & $0.522 * * *$ & $479.487 * * *$ & $0.005 * *$ & $0.076 \mathrm{~ns}$ & $0.029 *$ \\
\hline $\begin{array}{l}\text { Treatment } \\
\text { time }(\mathrm{T})\end{array}$ & 2 & $32.765^{*}$ & $101.304^{* *}$ & $78.364 * * *$ & $3.789 * * *$ & $186.398 * * *$ & $0.765^{*}$ & $0.345^{* *}$ & $0.0523^{*}$ \\
\hline $\begin{array}{l}\text { Cultivars } \\
\text { (Cv) }\end{array}$ & 3 & $1379.752 * * *$ & $485.440 * * *$ & $626.596 * *$ & $6.256 * * *$ & $8236.089 * * *$ & $0.076^{* *}$ & $0.150 * *$ & $1.150 * *$ \\
\hline $\begin{array}{c}\text { Interactions } \\
\text { Rip x T }\end{array}$ & 18 & $2050.401 *$ & $9870.96^{* *}$ & $4115.991 * * *$ & $1.977858 * *$ & $89375.42 * * *$ & $0.0038^{*}$ & $0.0262 \mathrm{~ns}$ & $0.0015^{*}$ \\
\hline Rip x Cv & 27 & $86343.5^{* *}$ & $47300.79 * * *$ & $32911.33^{* * * *}$ & $3.265 * * *$ & $3949098 * * *$ & $0.00038^{* *}$ & 0.0114* & 0.0333* \\
\hline $\mathrm{T} \times \mathrm{Cv}$ & 6 & $45207.57^{*}$ & $49177.01 * *$ & 49102.57 *** & $23.703 * *$ & $1535191 * * *$ & $0.0581^{*}$ & $0.0517 *$ & $0.0601 *$ \\
\hline Rip x T x Cv & 54 & $2829045^{*}$ & $4791759 * *$ & $2579063^{* *}$ & $12.373^{* * *}$ & $7386722 * *$ & $0.00029^{*}$ & $0.0039^{*}$ & $0.0017 *$ \\
\hline Error & 40 & 4237.943 & 2346.421 & 452.614 & 74.043 & 103.011 & 1.973 & 0.967 & 0.456 \\
\hline Total & 159 & & & & & & & & \\
\hline
\end{tabular}

\section{Sugar yield $\left(\mathrm{t} \mathrm{ha}^{-1}\right)$ of planted crop}

Sugar yield was highly significantly affected by the applications of chemical ripeners. Sugar yield increased in all the cultivars by the treatments of chemical ripeners. Effect of chemical ripeners for cultivars and their interactions (ripeners $\mathrm{x}$ cultivar) also yielded highly significant results (Table 1). Higher sugar yield was obtained from NSG-555 and the lowest sugar yield was calculated in HSF-242. Higher sugar yield was noted by the applications of $200 \mathrm{ppm}$ Sulfometuron-methyl and Glyphosate that was applied 45 days before cane harvesting. All the chemical ripeners applied 45 days before cane harvesting were the best for the production of sugar as compared to other treatments (Fig. 2B). As the chemical ripeners affected the juice extraction, juice purity and sugar recovery $\%$ of cane that resulted higher production of sugar (Table 2).

\section{Antioxidant activities in planted crop}

Antioxidant activities i.e. peroxidase (POD), catalases (CAT) and superoxide dismutase (SOD) were determined by evaluating the effects of Ethephon, Glyphosate and Sulfometuron-methyl. Effect of chemical ripeners was significant on POD activities in sugarcane (Table 1). Higher POD activities were noted in cultivar HSF-242 and the lowest was noted in HSF-240 (Fig. 3A). Maximum changes in POD was noted by the applications of Ethephon that were applied 30 and 45 days before cane harvesting. Cultivar HSF-240 had higher POD activities for Sulfometuron-methyl applications. There was a non-significant effect of chemical ripeners for CAT activities, however there were significant variations among cultivars (Table 1). Higher values of CAT were noted in HSF-240 and the lowest in cultivar HSF-242 (Fig. 3B). SOD activities were significantly changed by Chemical ripeners (Table 2). Maximum variations were noted in HSF-240 and the lowest values were noted in HSF-242 (Fig. 3C). Maximum value of 
SOD was noted by the treatment of Glyphosate that was applied 45 days before cane harvesting.

\section{Ratoon sprouting (\%)}

Data for ratoon sprouting of sugarcane crop is given in Table 3. Effect of chemical ripeners was non-significant on ratoon sprouting, however there was significant variations among cultivars. High sprouting \% was noted in NSG-555 and the lowest in HSF-242 (Fig. 4A). Ethephon treatment applied 45 days before cane harvesting were the best as compared to other treatments.

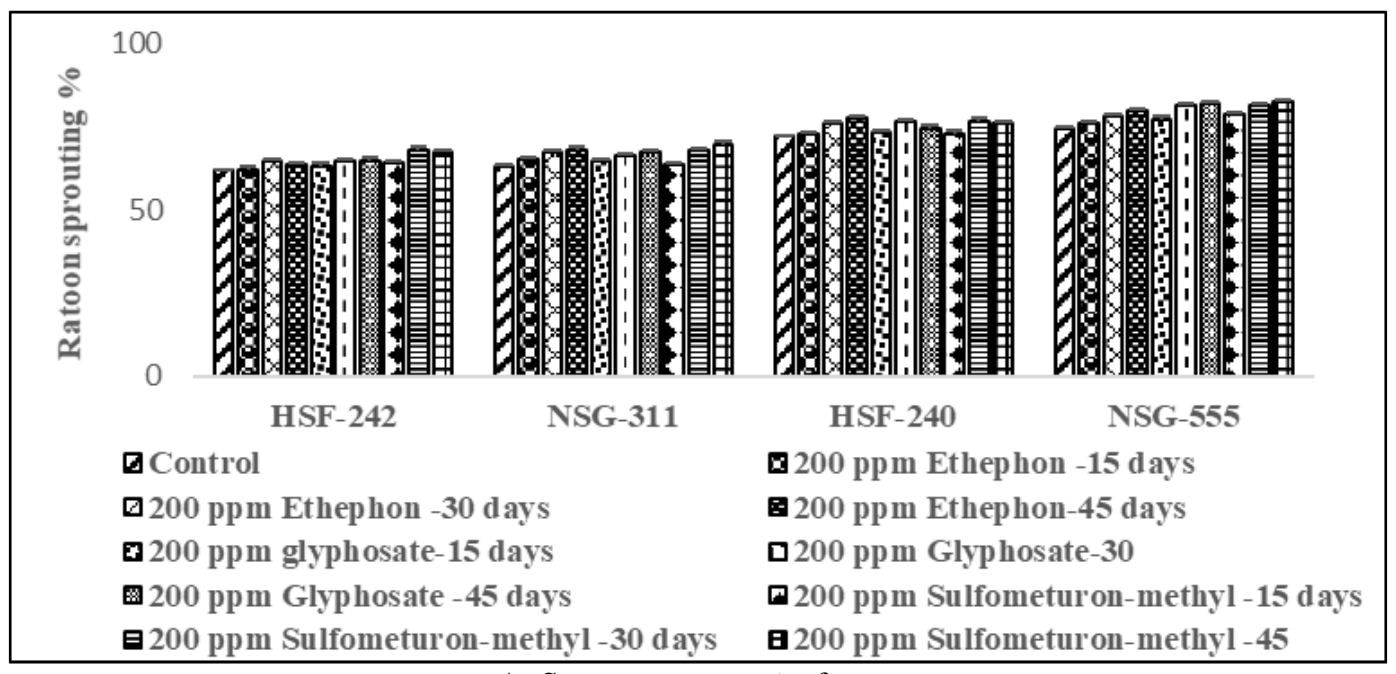

A: Sugar recovery $\%$ of cane

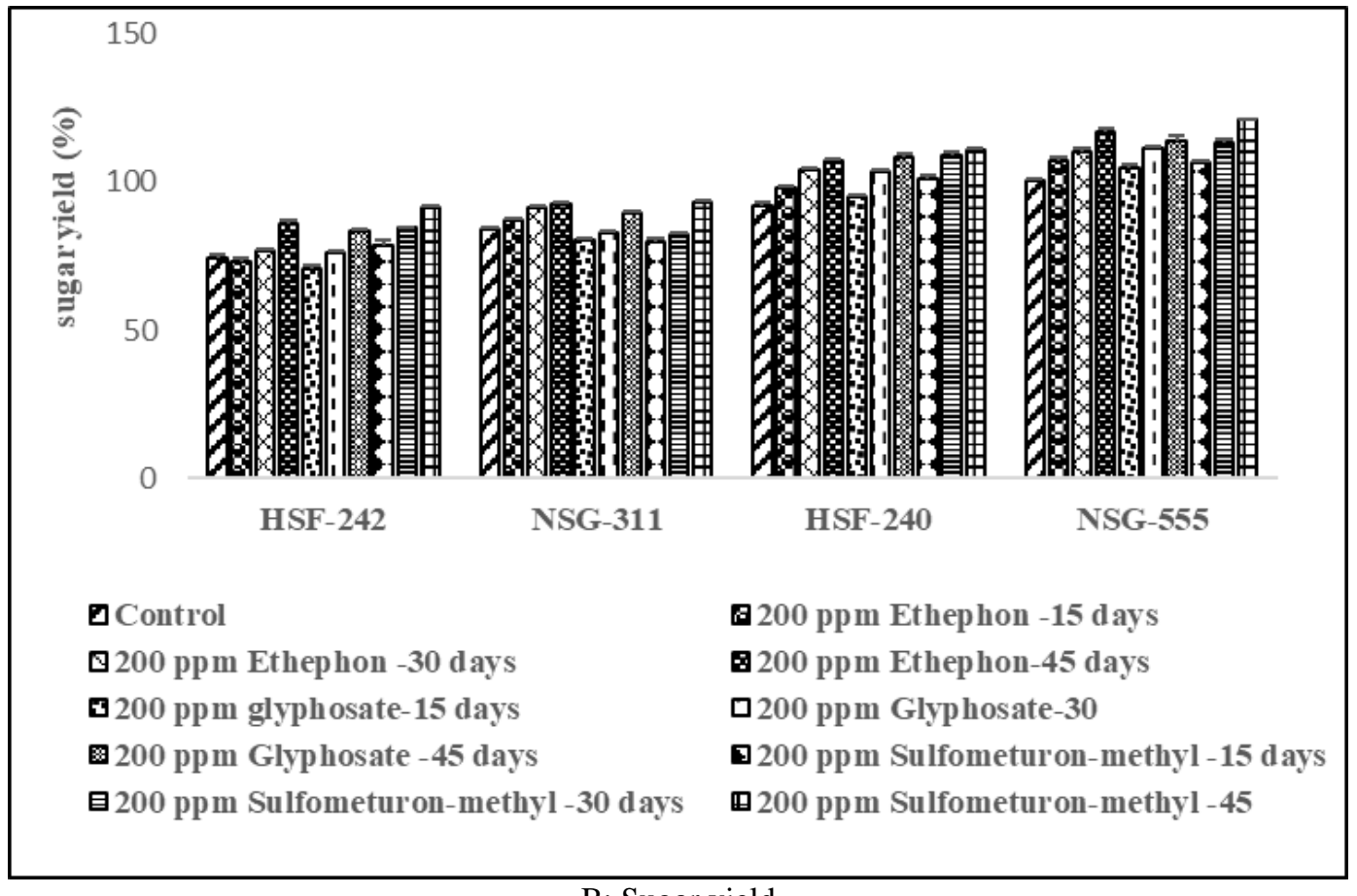

B: Sugar yield

Figure 2. Effect of different chemical ripeners on sugar yielding attributes of sugarcane cultivars in planted crop 
Table 2. Mean comparison of significant results using Tukey's test for different variable of sugarcane cultivars in planted crop

\begin{tabular}{|c|c|c|c|c|c|c|c|c|c|c|}
\hline Cultivar & Treatments & $\begin{array}{c}\text { Harvest index } \\
\%\end{array}$ & $\begin{array}{c}\text { Juice extraction } \\
\%\end{array}$ & $\begin{array}{c}\text { Juice purity } \\
\%\end{array}$ & $\begin{array}{c}\text { Pol } \\
\%\end{array}$ & $\begin{array}{c}\text { Sugar recovery } \\
\%\end{array}$ & $\begin{array}{c}\text { Sugar yield } \\
\left(\mathrm{t} \mathrm{ha}^{-1}\right)\end{array}$ & $\begin{array}{c}\text { Peroxidase } \\
\text { (POD) }\end{array}$ & $\begin{array}{c}\text { Catalases } \\
\text { (CAT) }\end{array}$ & \begin{tabular}{|c|} 
Superoxide \\
dismutase (SOD)
\end{tabular} \\
\hline \multirow{10}{*}{ HSF-242 } & Control & $65.62 \pm 1.2 \mathrm{BC}$ & $51.22 \pm 0.99 \mathrm{C}$ & $71.82 \pm 2.1 \mathrm{BC}$ & $14.21 \pm 1.1 \mathrm{D}$ & $9.12 \pm 0.91 \mathrm{E}$ & $74.32 \pm 3.4 \mathrm{D}$ & $0.892 \pm 0.03 \mathrm{C}$ & $1.027 \pm 0.04 \mathrm{~A}$ & $0.665 \pm 0.02 \mathrm{C}$ \\
\hline & Ethephon (15 days) & $65.61 \pm 2.2 \mathrm{BC}$ & $57.35 \pm 1.2 \mathrm{~B}$ & $71.57 \pm 2.4 \mathrm{BC}$ & $14.66 \pm 1.7 \mathrm{D}$ & $9.97 \pm 0.76 \mathrm{CD}$ & $72.91 \pm 2.8 \mathrm{D}$ & $0.895 \pm 0.01 \mathrm{C}$ & $1.037 \pm 0.03 \mathrm{~A}$ & $0.667 \pm 0.02 \mathrm{C}$ \\
\hline & Ethephon (30 days) & $65.41 \pm 1.9 \mathrm{BC}$ & $63.11 \pm 2.3 \mathrm{~A}$ & $74.05 \pm 1.9 \mathrm{~B}$ & $15.19 \pm 0.98 \mathrm{C}$ & $10.19 \pm 0.97 \mathrm{C}$ & $76.81 \pm 4.2 \mathrm{C}$ & $0.912 \pm 0.04 \mathrm{~B}$ & $1.025 \pm 0.04 \mathrm{~A}$ & $0.721 \pm 0.01 \mathrm{~B}$ \\
\hline & Ethephon (45 days) & $71.15 \pm 1.4 \mathrm{~A}$ & $67.85 \pm 2.2 \mathrm{~A}$ & $76.06 \pm 2.4 \mathrm{~A}$ & $16.51 \pm 1.2 \mathrm{~B}$ & $11.23 \pm 1.01 \mathrm{~A}$ & $86.05 \pm 4.4 \mathrm{AB}$ & $0.991 \pm 0.04 \mathrm{~B}$ & $1.042 \pm 0.06 \mathrm{~A}$ & $0.742 \pm 0.04 \mathrm{~A}$ \\
\hline & Glyphosate (15 days) & $66.17 \pm 2.1 \mathrm{~B}$ & $56.32 \pm 1.7 \mathrm{~B}$ & $71.97 \pm 1.7 \mathrm{BC}$ & $16.54 \pm 1.6 \mathrm{~A}$ & $10.21 \pm 0.92 \mathrm{BC}$ & $70.87 \pm 2.5 \mathrm{D}$ & $1.010 \pm 0.02 \mathrm{~A}$ & $1.051 \pm 0.03 \mathrm{~A}$ & $0.665 \pm 0.04 \mathrm{C}$ \\
\hline & Glyphosate (30 days) & $68.57 \pm 3.1 \mathrm{~B}$ & $56.01 \pm 1.8 \mathrm{~B}$ & $72.82 \pm 2.1 \mathrm{~B}$ & $16.68 \pm 1.7 \mathrm{~B}$ & $10.39 \pm 0.73 \mathrm{BC}$ & $75.98 \pm 2.1 \mathrm{C}$ & $1.007 \pm 0.03 \mathrm{~A}$ & $1.061 \pm 0.04 \mathrm{~A}$ & $0.712 \pm 0.03 \mathrm{~B}$ \\
\hline & Glyphosate (45 days) & $73.81 \pm 2.7 \mathrm{~A}$ & $60.47 \pm 2.0 \mathrm{~B}$ & $78.55 \pm 2.7 \mathrm{~A}$ & $16.93 \pm 0.72 \mathrm{~A}$ & $10.51 \pm 0.72 \mathrm{~B}$ & $83.63 \pm 3.4 \mathrm{~B}$ & $1.012 \pm 0.04 \mathrm{~A}$ & $1.034 \pm 0.01 \mathrm{~A}$ & $0.752 \pm 0.02 \mathrm{~A}$ \\
\hline & $\begin{array}{c}\text { Sulfometuron-methyl } \\
\text { (15 days) }\end{array}$ & $68.65 \pm 3.1 \mathrm{~B}$ & $53.05 \pm 1.1 \mathrm{C}$ & $70.55 \pm 1.8 \mathrm{C}$ & $16.29 \pm 1.7 \mathrm{BC}$ & $10.06 \pm 0.88 \mathrm{C}$ & $78.83 \pm 2.2 \mathrm{C}$ & $1.060 \pm 0.02 \mathrm{~A}$ & $1.067 \pm 0.04 \mathrm{~A}$ & $0.677 \pm 0.05 \mathrm{C}$ \\
\hline & $\begin{array}{c}\text { Sulfometuron-methyl } \\
\text { (30 days) }\end{array}$ & $67.87 \pm 1.4 \mathrm{~B}$ & $55.35 \pm 1.5 \mathrm{C}$ & $73.31 \pm 1.4 \mathrm{~B}$ & $16.44 \pm 1.5 \mathrm{~B}$ & $10.25 \pm 0.54 \mathrm{BC}$ & $84.41 \pm 2.8 \mathrm{~B}$ & $1.037 \pm 0.03 \mathrm{~A}$ & $1.064 \pm 0.03 \mathrm{~A}$ & $0.714 \pm 0.06 \mathrm{~B}$ \\
\hline & $\begin{array}{c}\text { Sulfometuron-methyl } \\
\text { (45 days) }\end{array}$ & $76.22 \pm 2.2 \mathrm{~A}$ & $63.82 \pm 2.3 \mathrm{~A}$ & $75.34 \pm 2.1 \mathrm{~A}$ & $17.02 \pm 1.9 \mathrm{~A}$ & $10.99 \pm 0.62 \mathrm{AB}$ & $91.41 \pm 3.8 \mathrm{~A}$ & $1.061 \pm 0.04 \mathrm{~A}$ & $1.072 \pm 0.05 \mathrm{~A}$ & $0.732 \pm 0.02 \mathrm{~A}$ \\
\hline \multirow{10}{*}{ NSG-311 } & Control & $58.27 \pm 1.0 \mathrm{C}$ & $57.92 \pm 1.3 \mathrm{~B}$ & $77.25 \pm 3.2 \mathrm{~A}$ & $14.06 \pm 1.5 \mathrm{D}$ & $9.06 \pm 0.43 \mathrm{D}$ & $83.76 \pm 3.8 \mathrm{~B}$ & $0.996 \pm 0.03 \mathrm{~B}$ & $1.102 \pm 0.04 \mathrm{~B}$ & $0.815 \pm 0.03 \mathrm{BC}$ \\
\hline & Ethephon (15 days) & $60.17 \pm 1.3 \mathrm{C}$ & $59.35 \pm 1.2 \mathrm{~B}$ & $73.97 \pm 2.9 \mathrm{BC}$ & $15.16 \pm 0.99 \mathrm{C}$ & $10.16 \pm 0.76 \mathrm{BC}$ & $86.82 \pm 3.9 \mathrm{AB}$ & $0.967 \pm 0.04 \mathrm{~B}$ & $1.092 \pm 0.03 \mathrm{~B}$ & $0.835 \pm 0.04 \mathrm{~B}$ \\
\hline & Ethephon (30 days) & $58.75 \pm 1.6 \mathrm{C}$ & $61.51 \pm 2.1 \mathrm{~B}$ & $74.77 \pm 3.5 \mathrm{~B}$ & $16.30 \pm 1.1 \mathrm{~B}$ & $10.30 \pm 0.73 \mathrm{~B}$ & $91.15 \pm 4.3 \mathrm{~A}$ & $1.011 \pm 0.05 \mathrm{~A}$ & $1.107 \pm 0.06 \mathrm{~A}$ & $0.869 \pm 0.03 \mathrm{~A}$ \\
\hline & Ethephon (45 days) & $72.55 \pm 2.3 \mathrm{~A}$ & $66.93 \pm 1.3 \mathrm{~A}$ & $75.55 \pm 2.6 \mathrm{~B}$ & $16.40 \pm 0.94 \mathrm{~B}$ & $11.40 \pm 0.92 \mathrm{AB}$ & $92.29 \pm 3.9 \mathrm{~A}$ & $1.015 \pm 0.05 \mathrm{~A}$ & $1.101 \pm 0.02 \mathrm{~A}$ & $0.887 \pm 0.03 \mathrm{~A}$ \\
\hline & Glyphosate (15 days) & $62.42 \pm 1.1 \mathrm{~B}$ & $56.32 \pm 0.92 \mathrm{C}$ & $74.21 \pm 1.8 \mathrm{~B}$ & $15.24 \pm 1.2 \mathrm{C}$ & $10.24 \pm 0.84 \mathrm{~B}$ & $80.28 \pm 3.4 \mathrm{BC}$ & $0.895 \pm 0.03 \mathrm{C}$ & $1.102 \pm 0.04 \mathrm{~B}$ & $0.841 \pm 0.05 \mathrm{~B}$ \\
\hline & Glyphosate (30 days) & $61.45 \pm 1.4 \mathrm{~B}$ & $59.97 \pm \mathrm{B}$ & $77.12 \pm 2.3 \mathrm{~A}$ & $15.36 \pm 1.3 \mathrm{C}$ & $10.36 \pm 0.34 \mathrm{~B}$ & $82.56 \pm 2.8 \mathrm{~B}$ & $1.017 \pm 0.04 \mathrm{~A}$ & $1.107 \pm 0.03 \mathrm{~A}$ & $0.872 \pm 0.04 \mathrm{~A}$ \\
\hline & Glyphosate (45 days) & $75.61 \pm 2.4 \mathrm{~A}$ & $61.82 \pm 1.1 \mathrm{~B}$ & $80.02 \pm 3.3 \mathrm{~A}$ & $16.40 \pm 1.5 \mathrm{~B}$ & $10.40 \pm 0.56 \mathrm{~B}$ & $89.69 \pm 3.3 \mathrm{~A}$ & $1.027 \pm 0.03 \mathrm{~A}$ & $1.112 \pm 0.08 \mathrm{~A}$ & $0.875 \pm 0.04 \mathrm{~A}$ \\
\hline & $\begin{array}{c}\text { Sulfometuron-methyl } \\
\text { (15 days) }\end{array}$ & $60.65 \pm 1.7 \mathrm{~B}$ & $55.25 \pm 0.82 \mathrm{C}$ & $72.82 \pm 2.2 \mathrm{C}$ & $15.16 \pm 0.99 \mathrm{C}$ & $10.16 \pm 0.74 \mathrm{BC}$ & $80.05 \pm 4.1 \mathrm{BC}$ & $0.937 \pm 0.05 \mathrm{BC}$ & $1.115 \pm 0.03 \mathrm{~A}$ & $0.807 \pm 0.02 \mathrm{C}$ \\
\hline & $\begin{array}{c}\text { Sulfometuron-methyl } \\
\text { (30 days) }\end{array}$ & $61.41 \pm 2.6 \mathrm{~B}$ & $55.61 \pm 0.91 \mathrm{C}$ & $74.13 \pm 1.8 \mathrm{~B}$ & $16.32 \pm 1.3 \mathrm{~B}$ & $10.32 \pm 0.65 \mathrm{~B}$ & $82.17 \pm 3.7 \mathrm{~B}$ & $1.011 \pm 0.05 \mathrm{~A}$ & $1.117 \pm 0.06 \mathrm{~A}$ & $0.825 \pm 0.03 \mathrm{~B}$ \\
\hline & $\begin{array}{c}\text { Sulfometuron-methyl } \\
\text { (45 days) }\end{array}$ & $75.33 \pm 1.1 \mathrm{~A}$ & $61.97 \pm 1.1 \mathrm{~B}$ & $79.92 \pm 2.4 \mathrm{~A}$ & $17.10 \pm 1.8 \mathrm{~A}$ & $11.10 \pm 0.93 \mathrm{~A}$ & $92.97 \pm 4.1 \mathrm{~A}$ & $1.027 \pm 0.04 \mathrm{~A}$ & $1.121 \pm 0.05 \mathrm{~A}$ & $0.881 \pm 0.01 \mathrm{~A}$ \\
\hline \multirow{8}{*}{ HSF-240 } & Control & $72.82 \pm 1.9 \mathrm{~B}$ & $64.41 \pm 1.4 \mathrm{~A}$ & $79.02 \pm 1.9 \mathrm{AB}$ & $14.31 \pm 0.96 \mathrm{C}$ & $9.44 \pm 0.48 \mathrm{E}$ & $91.96 \pm 2.8 \mathrm{C}$ & $0.865 \pm 0.03 \mathrm{D}$ & $1.112 \pm 0.05 \mathrm{AB}$ & $0.907 \pm 0.05 \mathrm{CD}$ \\
\hline & Ethephon (15 days) & $73.37 \pm 2.2 \mathrm{~B}$ & $65.35 \pm 2.1 \mathrm{~A}$ & $80.71 \pm 2.8 \mathrm{~A}$ & $16.06 \pm 1.6 \mathrm{AB}$ & $10.98 \pm 1.12 \mathrm{~B}$ & $97.73 \pm 5.2 \mathrm{~B}$ & $0.876 \pm 0.04 \mathrm{D}$ & $1.131 \pm 0.07 \mathrm{~A}$ & $0.932 \pm 0.04 \mathrm{~B}$ \\
\hline & Ethephon (30 days) & $72.02 \pm 1.6 \mathrm{~B}$ & $66.05 \pm 2.2 \mathrm{~A}$ & $80.95 \pm 3.2 \mathrm{~A}$ & $15.64 \pm 1.1 \mathrm{~B}$ & $10.70 \pm 0.74 \mathrm{~B}$ & $103.88 \pm 4.8 \mathrm{~A}$ & $0.875 \pm 0.02 \mathrm{D}$ & $1.137 \pm 0.08 \mathrm{~A}$ & $0.907 \pm 0.06 \mathrm{~B}$ \\
\hline & Ethephon (45 days) & $83.45 \pm 3.2 \mathrm{~A}$ & $61.57 \pm 3.1 \mathrm{~B}$ & $82.95 \pm 2.7 \mathrm{~A}$ & $16.99 \pm 1.4 \mathrm{~A}$ & $11.67 \pm 0.86 \mathrm{~A}$ & $107.36 \pm 5.4 \mathrm{~A}$ & $0.935 \pm 0.02 \mathrm{~B}$ & $1.132 \pm 0.03 \mathrm{~A}$ & $1.011 \pm 0.03 \mathrm{~A}$ \\
\hline & Glyphosate (15 days) & $72.65 \pm 2.8 \mathrm{~B}$ & $63.22 \pm 2.4 \mathrm{~B}$ & $81.05 \pm 1.7 \mathrm{~A}$ & $14.52 \pm 0.89 \mathrm{C}$ & $10.34 \pm 0.44 \mathrm{D}$ & $94.84 \pm 3.9 \mathrm{~B}$ & $0.941 \pm 0.01 \mathrm{~B}$ & $1.151 \pm 0.05 \mathrm{~A}$ & $0.917 \pm 0.04 \mathrm{~B}$ \\
\hline & Glyphosate (30 days) & $70.02 \pm 1.7 \mathrm{C}$ & $65.17 \pm 1.8 \mathrm{~A}$ & $81.75 \pm 2.8 \mathrm{~A}$ & $15.69 \pm 0.99 \mathrm{~B}$ & $10.48 \pm 0.56 \mathrm{C}$ & $103.45 \pm 4.2 \mathrm{~A}$ & $0.950 \pm 0.03 \mathrm{~B}$ & $1.135 \pm 0.05 \mathrm{~A}$ & $1.015 \pm 0.04 \mathrm{~A}$ \\
\hline & Glyphosate (45 days) & $81.02 \pm 2.2 \mathrm{~A}$ & $66.72 \pm 1.1 \mathrm{~A}$ & $83.82 \pm 3.2 \mathrm{~A}$ & $14.81 \pm 1.3 \mathrm{C}$ & $10.61 \pm 0.73 \mathrm{C}$ & $108.50 \pm 5.6 \mathrm{~A}$ & $0.901 \pm 0.04 \mathrm{C}$ & $1.153 \pm 0.06 \mathrm{~A}$ & $1.115 \pm 0.05 \mathrm{~A}$ \\
\hline & $\begin{array}{c}\text { Sulfometuron-methyl } \\
\text { (15 days) }\end{array}$ & $69.85 \pm 1.8 \mathrm{C}$ & $59.61 \pm 0.91 \mathrm{BC}$ & $75.32 \pm 3.1 \mathrm{~B}$ & $15.21 \pm 1.1 \mathrm{BC}$ & $10.35 \pm 0.81 \mathrm{D}$ & $101.23 \pm 3.8 \mathrm{~A}$ & $1.001 \pm 0.04 \mathrm{~A}$ & $1.135 \pm 0.05 \mathrm{~A}$ & $0.857 \pm 0.04 \mathrm{C}$ \\
\hline
\end{tabular}

APPLIED ECOLOGY AND ENVIRONMENTAL RESEARCH 18(5):6405-6423.

http://www.aloki.hu • ISSN 15891623 (Print) • ISSN 17850037 (Online)

DOI: http://dx.doi.org/10.15666/aeer/1805_64056423

( 2020 , ALÖKI Kft., Budapest, Hungary 


\begin{tabular}{|c|c|c|c|c|c|c|c|c|c|c|}
\hline & $\begin{array}{c}\text { Sulfometuron-methyl } \\
\text { (30 days) }\end{array}$ & $70.52 \pm 2.2 \mathrm{C}$ & $62.02 \pm 1.1 \mathrm{~B}$ & $75.52 \pm 2.2 \mathrm{~B}$ & $16.22 \pm 1.3 \mathrm{~A}$ & $11.10 \pm 0.93 \mathrm{AB}$ & $108.77 \pm 5.5 \mathrm{~A}$ & $0.977 \pm 0.03 \mathrm{~B}$ & $1.112 \pm 0.03 \mathrm{~B}$ & $1.101 \pm 0.03 \mathrm{~A}$ \\
\hline & $\begin{array}{c}\text { Sulfometuron-methyl } \\
\text { (45 days) }\end{array}$ & $82.07 \pm 3.1 \mathrm{~A}$ & $66.37 \pm 1.0 \mathrm{~A}$ & $81.15 \pm 3.4 \mathrm{~A}$ & $16.56 \pm 1.6 \mathrm{~A}$ & $11.23 \pm 0.99 \mathrm{~A}$ & $110.67 \pm 5.0 \mathrm{~A}$ & $1.031 \pm 0.04 \mathrm{~A}$ & $1.123 \pm 0.04 \mathrm{~B}$ & $1.061 \pm 0.06 \mathrm{~A}$ \\
\hline \multirow{10}{*}{ NSG-555 } & \begin{tabular}{|l} 
Control \\
\end{tabular} & $57.82 \pm 2.4 \mathrm{~B}$ & $66.82 \pm 2.1 \mathrm{~A}$ & $77.51 \pm 1.8 \mathrm{~B}$ & $15.22 \pm 1.2 \mathrm{C}$ & $10.01 \pm 0.43 \mathrm{D}$ & $100.26 \pm 4.7 \mathrm{C}$ & $0.796 \pm 0.02 \mathrm{D}$ & $1.123 \pm 0.03 \mathrm{~B}$ & $0.997 \pm 0.04 \mathrm{D}$ \\
\hline & Ethephon (15 days) & $59.21 \pm 1.5 \mathrm{~B}$ & $67.10 \pm 3.2 \mathrm{~A}$ & $81.25 \pm 2.5 \mathrm{~A}$ & $16.31 \pm 1.4 \mathrm{~B}$ & $11.45 \pm 0.82 \mathrm{~B}$ & $106.97 \pm 5.4 \mathrm{BC}$ & $0.806 \pm 0.03 \mathrm{D}$ & $1.141 \pm 0.06 \mathrm{~A}$ & $1.072 \pm 0.03 \mathrm{C}$ \\
\hline & Ethephon (30 days) & $59.87 \pm 1.1 \mathrm{~B}$ & $66.87 \pm 3.3 \mathrm{~A}$ & $81.91 \pm 3.4 \mathrm{~A}$ & $16.27 \pm 1.6 \mathrm{~B}$ & $11.13 \pm 0.77 \mathrm{BC}$ & $110.18 \pm 4.8 \mathrm{~B}$ & $0.812 \pm 0.02 \mathrm{D}$ & $1.132 \pm 0.07 \mathrm{AB}$ & $1.117 \pm 0.07 \mathrm{~B}$ \\
\hline & Ethephon (45 days) & $67.32 \pm 1.5 \mathrm{~A}$ & $64.65 \pm 1.4 \mathrm{~A}$ & $83.52 \pm 2.6 \mathrm{~A}$ & $17.21 \pm 1.9 \mathrm{~A}$ & $12.12 \pm 1.01 \mathrm{~A}$ & $116.85 \pm 5.6 \mathrm{~A}$ & $0.822 \pm 0.03 \mathrm{BC}$ & $1.161 \pm 0.02 \mathrm{~A}$ & $1.145 \pm 0.07 \mathrm{~A}$ \\
\hline & Glyphosate (15 days) & $58.85 \pm 2.2 \mathrm{~B}$ & $65.77 \pm 2.3 \mathrm{~A}$ & $82.72 \pm 2.9 \mathrm{~A}$ & $17.43 \pm 1.7 \mathrm{~A}$ & $11.66 \pm 0.94 \mathrm{~B}$ & $104.74 \pm 4.5 \mathrm{BC}$ & $0.841 \pm 0.04 \mathrm{C}$ & $1.132 \pm 0.06 \mathrm{AB}$ & $1.115 \pm 0.06 \mathrm{~B}$ \\
\hline & Glyphosate (30 days) & $57.42 \pm 2.1 \mathrm{~B}$ & $66.71 \pm 1.6 \mathrm{~A}$ & $83.67 \pm 1.9 \mathrm{~A}$ & $16.54 \pm 1.9 \mathrm{~B}$ & $10.76 \pm 0.82 \mathrm{C}$ & $111.02 \pm 4.3 \mathrm{~B}$ & $0.853 \pm 0.03 \mathrm{C}$ & $1.157 \pm 0.04 \mathrm{~A}$ & $1.147 \pm 0.05 \mathrm{~A}$ \\
\hline & Glyphosate (45 days) & $67.87 \pm 2.3 \mathrm{~A}$ & $65.97 \pm 2.4 \mathrm{~A}$ & $83.05 \pm 3.1 \mathrm{~A}$ & $17.63 \pm 2.1 \mathrm{~A}$ & $11.44 \pm 0.92 \mathrm{~B}$ & $113.95 \pm 5.9 \mathrm{~B}$ & $0.912 \pm 0.04 \mathrm{~A}$ & $1.152 \pm 0.05 \mathrm{~A}$ & $1.135 \pm 0.06 \mathrm{~A}$ \\
\hline & $\begin{array}{c}\text { Sulfometuron-methyl } \\
\text { (15 days) }\end{array}$ & $56.57 \pm 1.7 \mathrm{BC}$ & $64.12 \pm 3.4 \mathrm{~A}$ & $82.42 \pm 3.7 \mathrm{~A}$ & $15.22 \pm 1.4 \mathrm{C}$ & $10.69 \pm 0.74 \mathrm{C}$ & $106.68 \pm 4.3 \mathrm{BC}$ & $0.847 \pm 0.02 \mathrm{C}$ & $1.132 \pm 0.02 \mathrm{AB}$ & $1.045 \pm 0.07 \mathrm{C}$ \\
\hline & $\begin{array}{c}\text { Sulfometuron-methyl } \\
\text { (30 days) }\end{array}$ & $59.65 \pm 1.2 \mathrm{~B}$ & $64.17 \pm 2.8 \mathrm{~A}$ & $83.82 \pm 2.4 \mathrm{~A}$ & $16.18 \pm 1.5 \mathrm{~B}$ & $11.21 \pm 0.85 \mathrm{BC}$ & $113.08 \pm 3.9 \mathrm{~A}$ & $0.881 \pm 0.04 \mathrm{~B}$ & $1.123 \pm 0.03 \mathrm{~B}$ & $1.105 \pm 0.05 \mathrm{~B}$ \\
\hline & $\begin{array}{c}\text { Sulfometuron-methyl } \\
\text { (45 days) }\end{array}$ & $66.77 \pm 2.2 \mathrm{~A}$ & $65.52 \pm 1.8 \mathrm{~A}$ & $82.27 \pm 2.2 \mathrm{~A}$ & $17.52 \pm 1.4 \mathrm{~A}$ & $11.41 \pm 0.90 \mathrm{~B}$ & $120.73 \pm 4.9 \mathrm{~A}$ & $0.923 \pm 0.05 \mathrm{~A}$ & $1.133 \pm 0.04 \mathrm{AB}$ & $1.137 \pm 0.08 \mathrm{~A}$ \\
\hline
\end{tabular}

$\pm(\mathrm{SE})=$ Standard error

In a column, means with different capital letters are statistically significant as determined by Tukey's test at $\mathrm{P} \leq 0.05$

Table 3. Mean squares (MS) from the Analysis of Variance (ANOVA) for ratooning growth and yield attributes of sugarcane cultivars under the applications of chemical ripeners

\begin{tabular}{|c|c|c|c|c|c|}
\hline Source & df & Ratoon sprouting (\%) & No. of tillers/plant & Mill-able canes & Ratoon cane yield \\
\hline $\begin{array}{c}\text { Main effects } \\
\text { Ripeners (Rip) }\end{array}$ & 9 & $1536.016 \mathrm{~ns}$ & $2.091 \mathrm{~ns}$ & $1379.649 \mathrm{~ns}$ & $27199.380 \mathrm{~ns}$ \\
\hline Treatment time $(\mathrm{T})$ & 2 & $456.671 \mathrm{~ns}$ & $4.786 \mathrm{~ns}$ & $341.132 \mathrm{~ns}$ & $1421.872 \mathrm{~ns}$ \\
\hline Cultivars (Cv) & 3 & $193.219 *$ & $1.655 *$ & $384.404 * *$ & $4622.580 * *$ \\
\hline $\begin{array}{c}\text { Interactions } \\
\text { Rip x T }\end{array}$ & 18 & $67388.09 \mathrm{~ns}$ & $256.6953 \mathrm{~ns}$ & $291993.15 n s$ & $2148017 \mathrm{~ns}$ \\
\hline Rip x Cv & 27 & $296787.5^{*}$ & $3.460605 \mathrm{~ns}$ & $530342.6^{*}$ & $1260006 \mathrm{~ns}$ \\
\hline $\mathrm{T} \times \mathrm{Cv}$ & 6 & $8476.904 n s$ & $203.1711 \mathrm{~ns}$ & $81356.42 *$ & $3650593 \mathrm{~ns}$ \\
\hline Rip x T x Cv & 54 & $13020660 *$ & $424.8308 \mathrm{~ns}$ & $1120400^{*}$ & 997643ns \\
\hline Error & 40 & 8657.149 & 860.910 & 1087.413 & 5081.462 \\
\hline Total & 159 & & & & \\
\hline
\end{tabular}

ns $=$ non-significant and $*$, ** = significant at $\mathrm{P}<0.05,0.01$ probability levels 


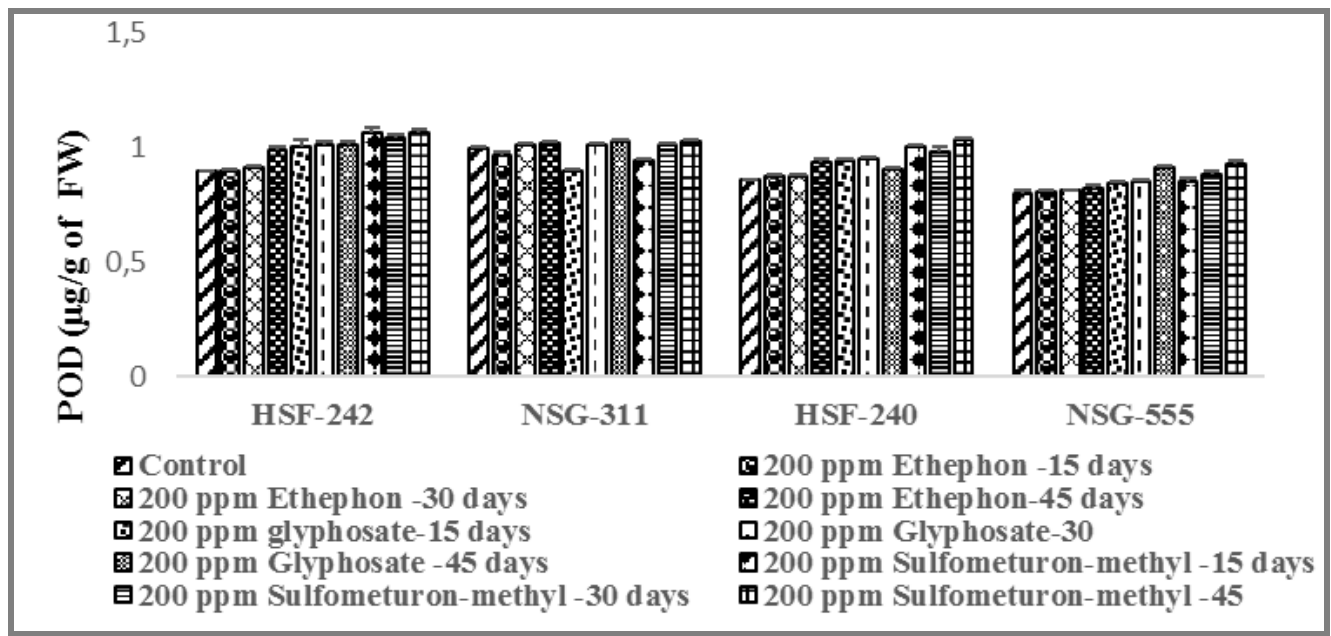

A: Peroxidase (POD)

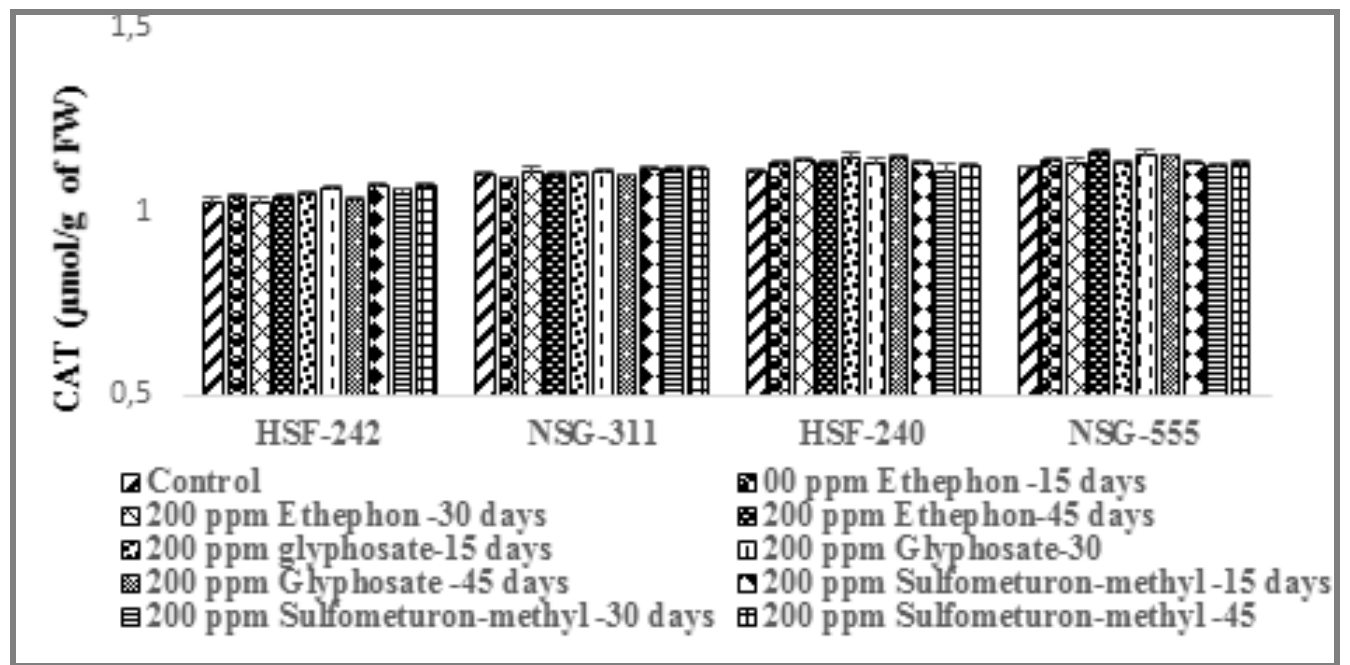

B: Catalases (CAT)

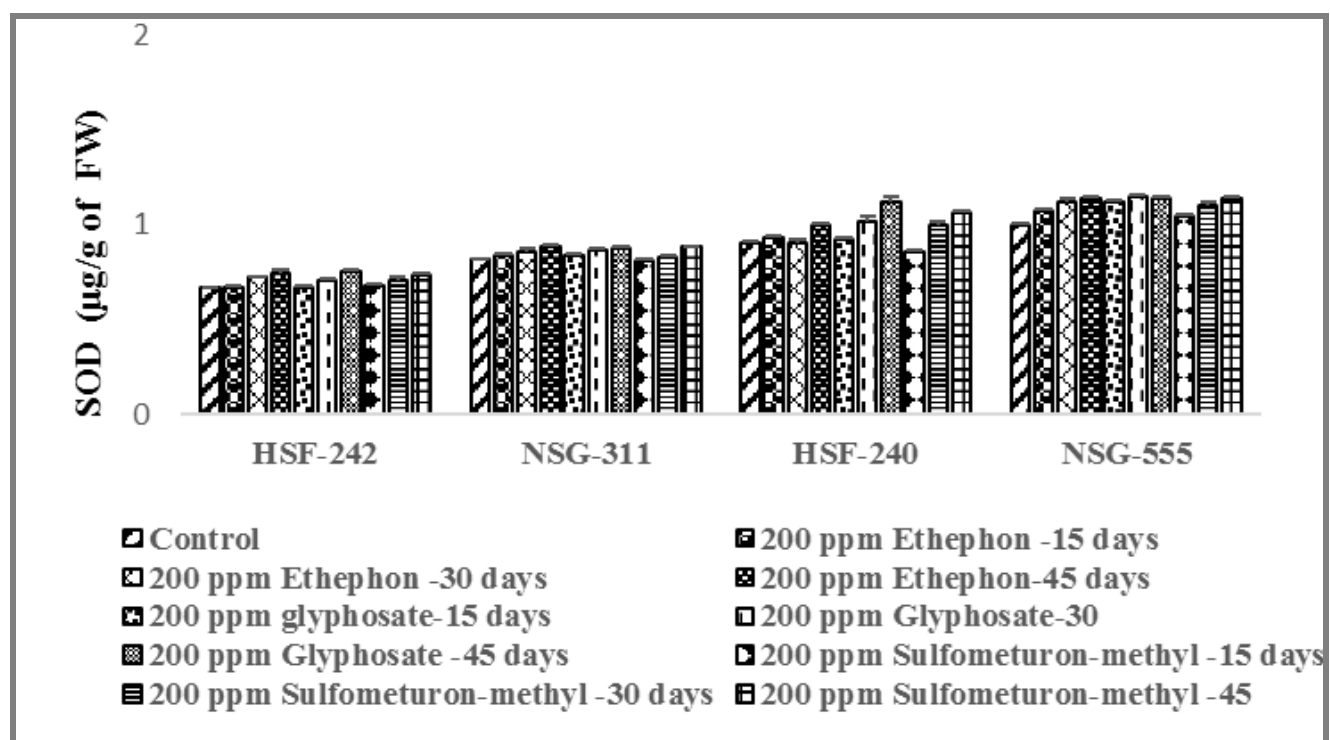

C: Superoxide dismutase (SOD)

Figure 3. Effect of different chemical ripeners on antioxidant activities of sugarcane cultivars in planted crop 


\section{No. of tillers/plant of ratooning}

Effect of chemical ripeners was non-significant on number of tillers/plant in ratooning crop, however there was significant variations among sugarcane cultivars (Table 3). Higher number of tillers was noted in NSG-555 cultivar and the lowest number of tillers was counted in HSF-242 (Fig. 4B).

\section{Number of millable canes of ratooning}

There was a non-significant effect of chemical ripeners on millable canes counted in ratooning crop (Table 3). Higher number of millable canes were counted in cultivar HSF-240 and the lowest number of millable cane was present in NSG-555 (Fig. 4C). Sulfometuron-methyl showed better results for millable canes in HSF-242.

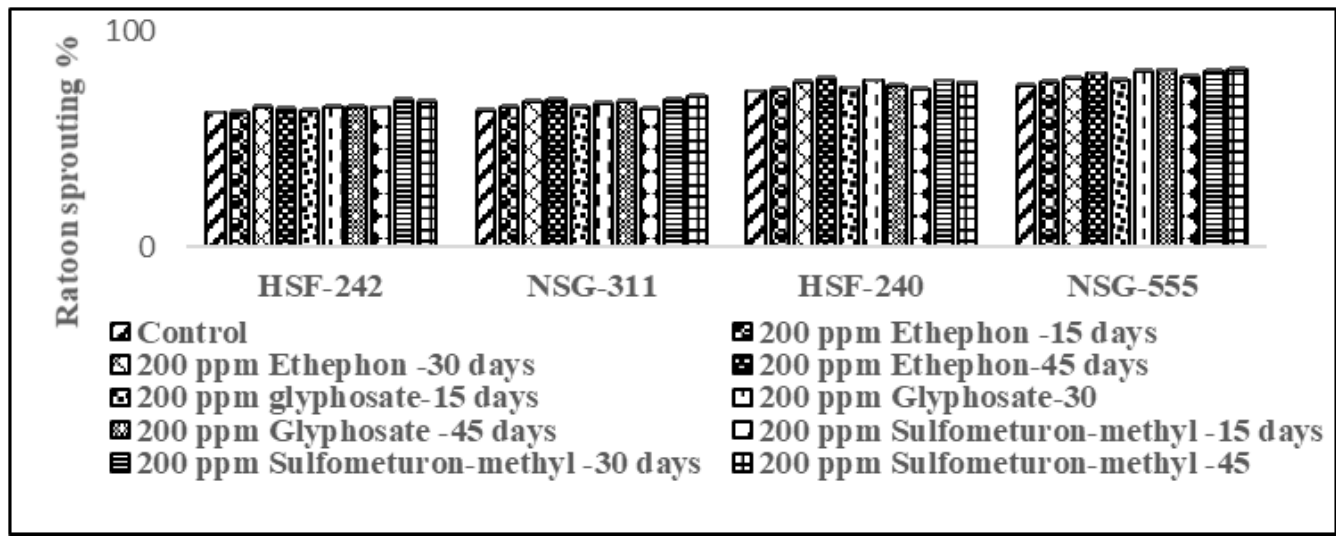

A: Ratoon sprouting

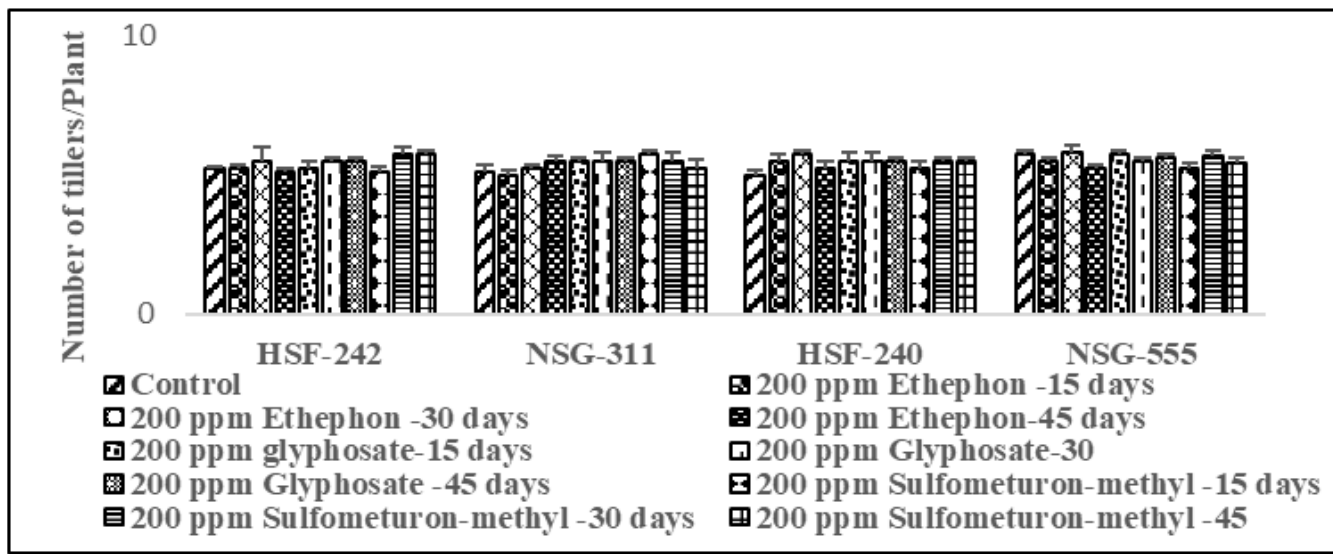

B: Number of tiller/plant

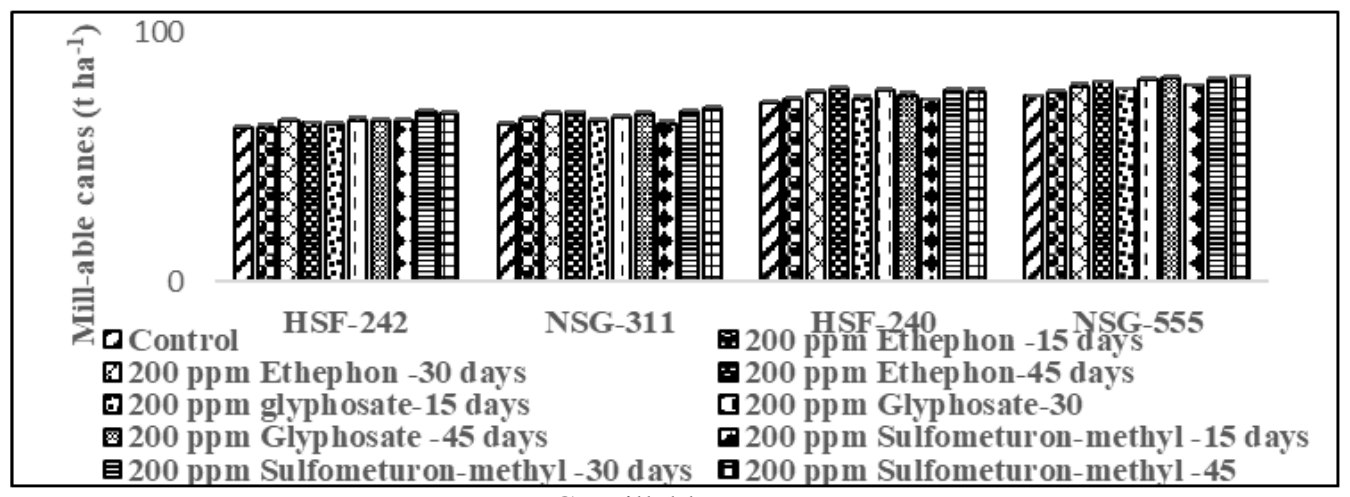

C: Millable canes 


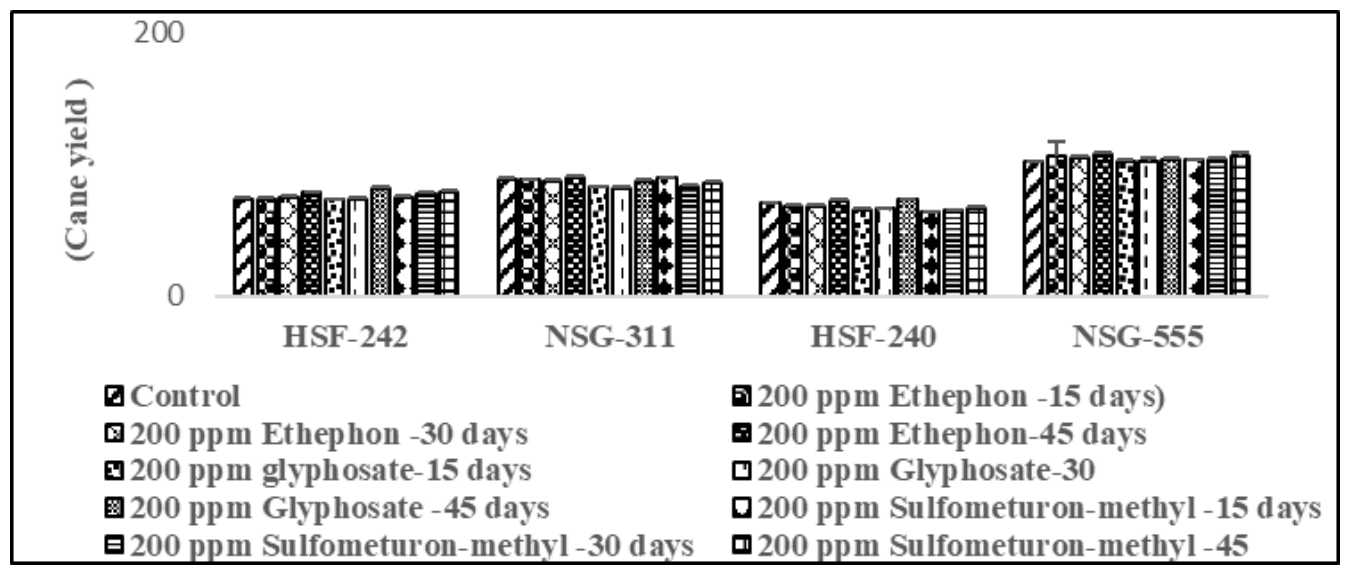

D: Cane yield

Figure 4. Effect of different chemical ripeners on ratooning growth and yield of sugarcane cultivars

\section{Cane yield $\left(\mathrm{tha}^{-1}\right)$ of ratooning}

Data for ratoon cane yield is given in Table 3. Effect of chemical ripeners was nonsignificant on cane yield, however there was significant variations among cultivars. Higher cane yield was calculated in NSG-555 and the lowest in HSF-240 (Fig. 4D). Chemical ripeners had non-significant effects on ratoon sprouting, number of tillers and millable canes due to that cane yield was also not affected.

\section{Juice extraction (\%) from ratooning}

Table 4 showed that there were non-significant results for juice extraction $\%$ for ratooning crop. It was apparent that chemical ripeners did not affect the growth and quality attributes. It only affected the quality attributes during the maturity of planted crop by the applications of chemical ripeners. HSF-240 cultivar was the best in juice extraction during ratooning harvest (Fig. 5A).

Table 4. Mean squares (MS) from the analysis of variance (ANOVA) for quality and antioxidant activities of ratooning sugarcane cultivars under the applications of chemical ripeners

\begin{tabular}{|c|c|c|c|c|c|c|c|c|}
\hline Source & df & $\begin{array}{c}\text { Juice } \\
\text { extraction }\end{array}$ & Juice purity & $\begin{array}{c}\text { Sugar } \\
\text { recovery } \% \\
\text { cane }\end{array}$ & $\begin{array}{c}\text { Total sugar } \\
\text { yield }\end{array}$ & $\begin{array}{l}\text { Peroxidase } \\
\text { (POD) } \\
\text { activities }\end{array}$ & $\begin{array}{l}\text { Catalases } \\
\text { (CAT) } \\
\text { activities }\end{array}$ & $\begin{array}{c}\text { Superoxide } \\
\text { dismutase } \\
\text { (SOD) } \\
\text { activities } \\
\end{array}$ \\
\hline $\begin{array}{l}\text { Main effects } \\
\text { Ripeners } \\
\text { (Rip) }\end{array}$ & 9 & $71.248 \mathrm{~ns}$ & $37.654^{*}$ & $68.934 \mathrm{~ns}$ & $49.694 \mathrm{~ns}$ & $0.065 \mathrm{~ns}$ & $0.0762 \mathrm{~ns}$ & $0.023 \mathrm{~ns}$ \\
\hline $\begin{array}{l}\text { Treatment } \\
\text { time }(\mathrm{T})\end{array}$ & 2 & $43.872 \mathrm{~ns}$ & $122.762 \mathrm{~ns}$ & $211.643 \mathrm{~ns}$ & $78.973 \mathrm{~ns}$ & $0.0342 \mathrm{~ns}$ & 0.0341 & $0.0642 \mathrm{~ns}$ \\
\hline Cultivars $(\mathrm{Cv})$ & 3 & $376.423 * *$ & $576.976^{* *} *$ & $387.459 *$ & $623.434 *$ & $1.561 *$ & $1.254 *$ & 1.150 ** \\
\hline $\begin{array}{c}\text { Interactions } \\
\text { Rip x T } \\
\end{array}$ & 18 & $3125.792 \mathrm{~ns}$ & $4622.48 \mathrm{~ns}$ & $14589.4 \mathrm{~ns}$ & $3924.484 \mathrm{~ns}$ & $0.0022 \mathrm{~ns}$ & $0.0025 \mathrm{~ns}$ & $0.0014 \mathrm{~ns}$ \\
\hline Rip x Cv & 27 & $26819.39 *$ & $21725.45 * *$ & $26709.1 *$ & $30980.93 *$ & $0.1014 \mathrm{~ns}$ & $0.0955 \mathrm{~ns}$ & $0.0264 \mathrm{~ns}$ \\
\hline $\mathrm{T} \times \mathrm{Cv}$ & 6 & $16514.43 \mathrm{~ns}$ & $70830.73^{*}$ & $82002.99 \mathrm{~ns}$ & $49234.45^{*}$ & $0.0533 \mathrm{~ns}$ & $0.042 \mathrm{~ns}$ & $0.0738 \mathrm{~ns}$ \\
\hline $\operatorname{Rip} \times \mathrm{T} \times \mathrm{Cv}$ & 54 & $117662 *$ & $2667060^{*}$ & $5652794 \mathrm{~ns}$ & $2446657 \mathrm{~ns}$ & $0.0034 \mathrm{~ns}$ & $0.0032 \mathrm{~ns}$ & $0.0016 \mathrm{~ns}$ \\
\hline Error & 40 & 234.324 & 265.764 & 558.695 & 333.421 & 1.057 & 0.778 & 0.634 \\
\hline Total & 159 & & & & & & & \\
\hline
\end{tabular}

$\mathrm{ns}=$ non-significant and $* * *=$ significant at $\mathrm{P}<0.05,0.01$ probability levels 


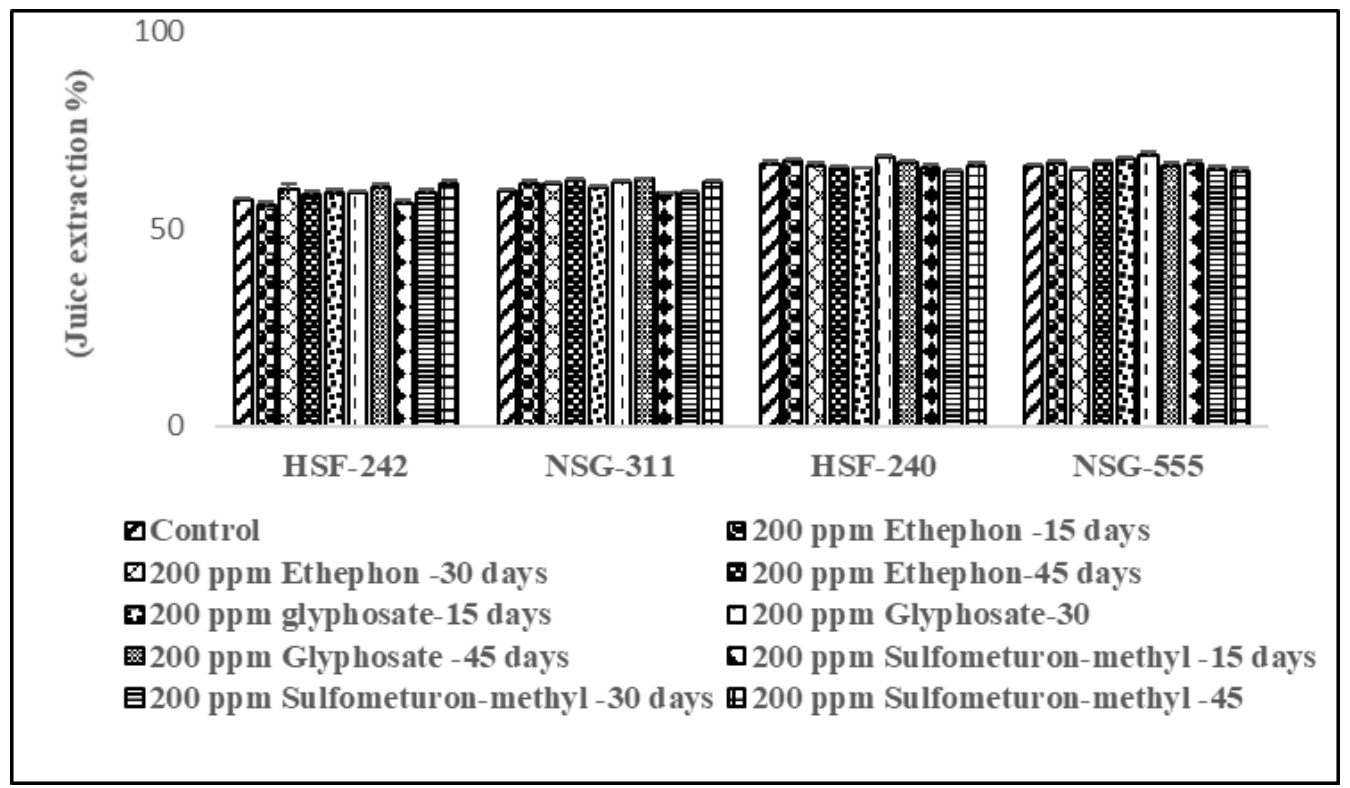

A: Juice extraction

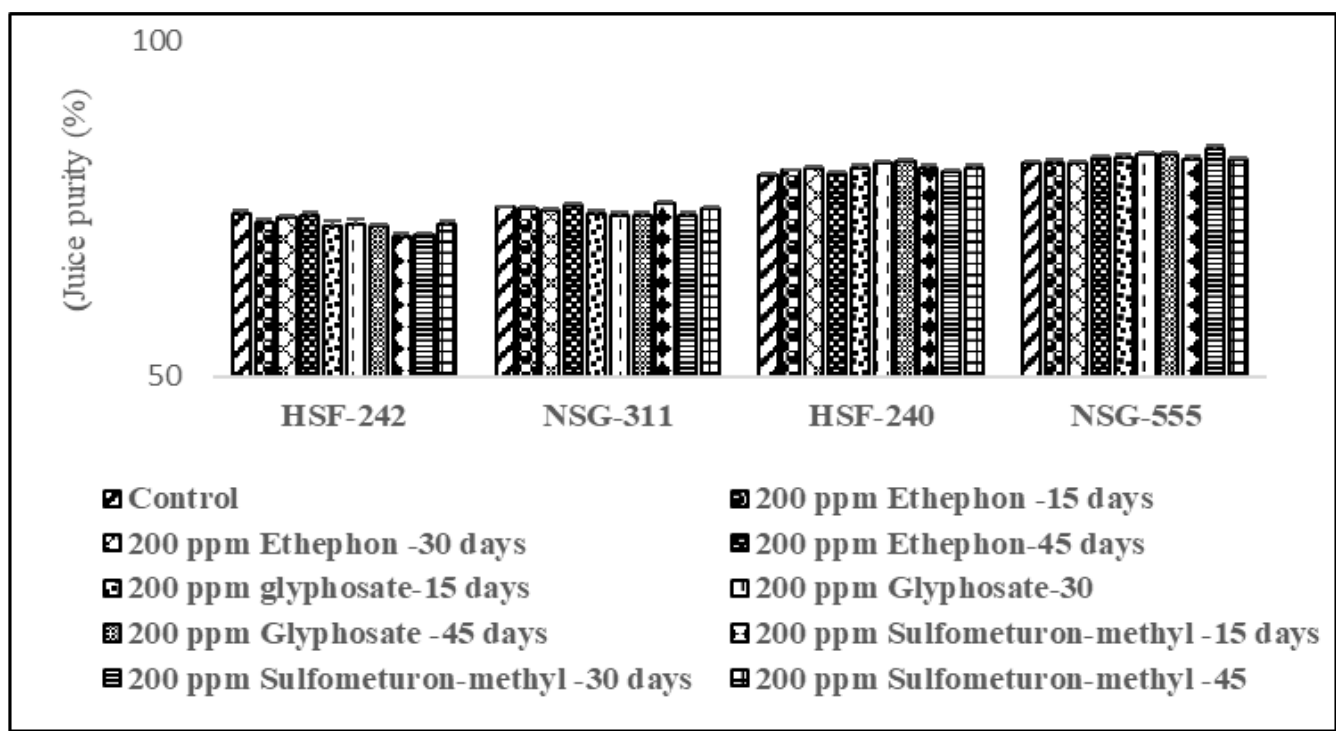

B: Juice purity

Figure 5. Effect of different chemical ripeners on juice extraction and purity of sugarcane cultivars in ratooning crop

\section{Juice purity (\%) in ratooning}

Effect of chemical ripeners was significant on juice purity \% for sugarcane (Table 4). HSF-242 showed the maximum variations for juice purity in response to chemical ripeners especially Ethephon applied 45 days before harvesting. There were also significant variations among cultivars in response to chemical ripeners. The highest juice purity \% was noted in NSG-555 and the lowest in HSF-242 (Fig. 5B).

\section{Sugar recovery \% of cane in ratooning}

There was a non-significant effect of chemical ripeners on sugar recovery $\%$ of cane (Table 4). Higher sugar recovery $\%$ of cane were calculated in cultivar NSG-555 and 
the lowest in HSF-242 (Fig. 6A). Results for sugar recovery \% of cane was nonsignificant as other quality related parameters were also non-significant that also resulted no change in sugar recovery $\%$ of cane.

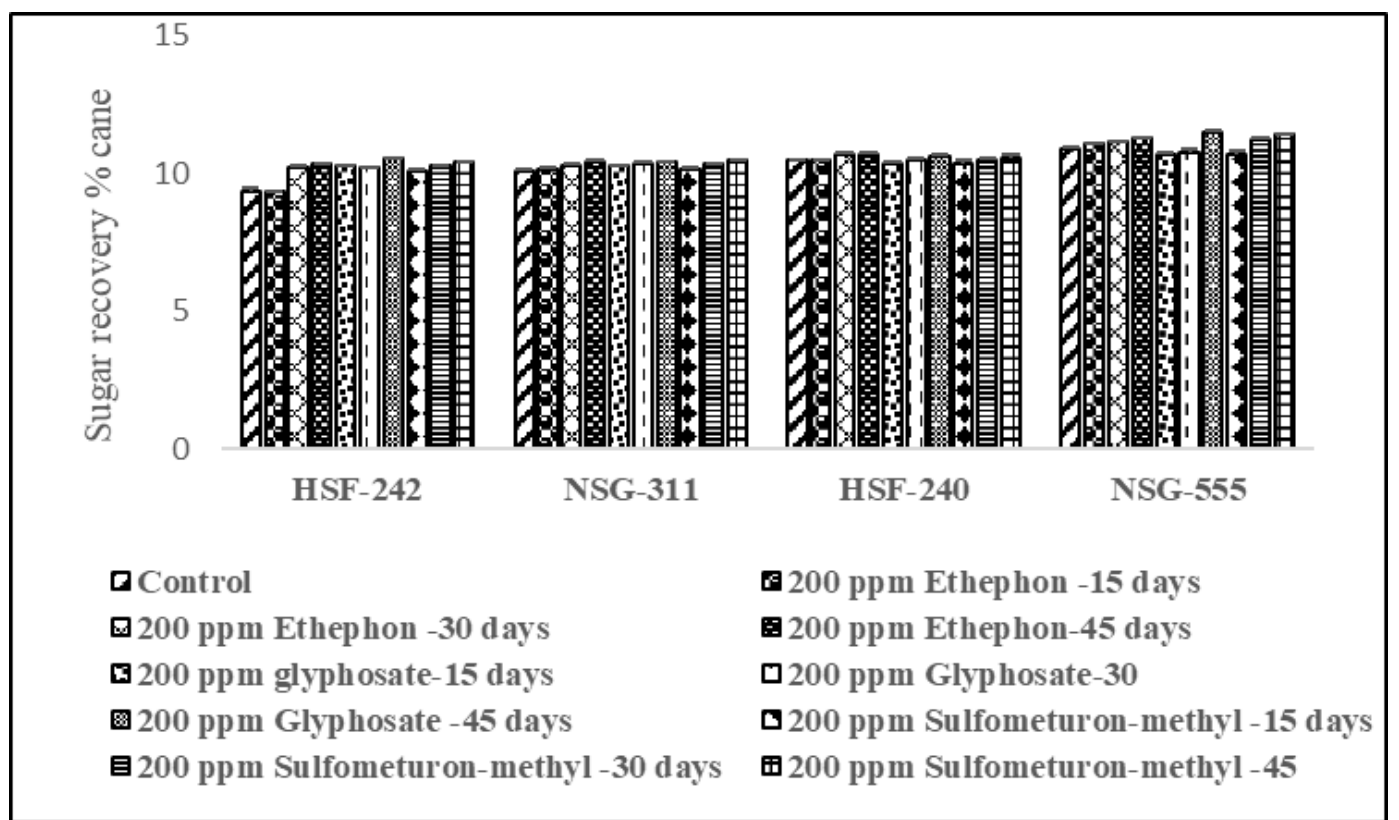

A: Sugar recovery $\%$ of cane

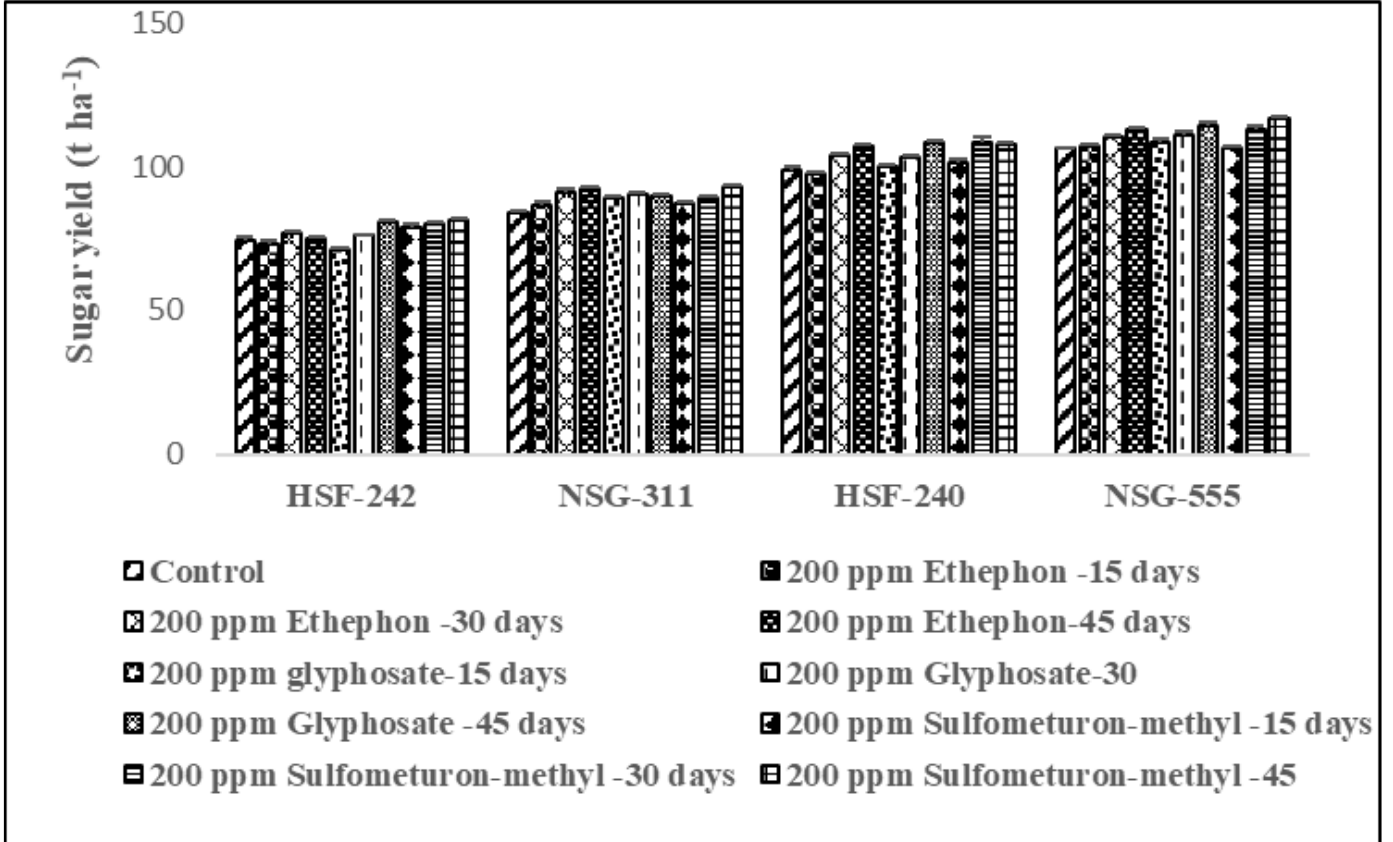

B: Sugar yield

Figure 6. Effect of different chemical ripeners on sugar yielding attributes of sugarcane cultivars in ratooning crop

\section{Total sugar yield ( $\left.\mathrm{t} \mathrm{ha}^{-1}\right)$ of ratooning}

Results for total sugar yield for ratooning crop was similar as in the case of sugar recovery \% of cane. There were non-significant results for total sugar yield (Table 4). 
Higher total sugar yield was calculated in cultivar NSG-555 and the lowest in HSF-242 (Fig. 6B). All the quality parameters had non-significant results for chemical ripeners that also resulted non-significant effects for total sugar yield.

\section{Antioxidant activities in ratooning}

There were non-significant effects for antioxidant activities i.e. peroxidase (POD), catalases (CAT) and superoxide dismutase (SOD). As on ratooning crop there was not any influence of chemical ripeners for growth, yield and quality that also affected antioxidant activities (Table 4). These results showed there was no change in ratooning crop towards any stimulant, its growth was normal depending upon the genetic makeup of sugarcane cultivars. Data related to antioxidant activities of POD, CAT and SOD is presented in Figure 7.

\section{Discussion}

Results have shown that chemical ripeners increased the harvest index (HI) of sugarcane. It might be due to the conversion of cane formation and elongation phase into ripening phase by Ethephon and Sulfometuron-methyl. Kapur et al. (2013) found the variations for $\mathrm{HI}$ in sugarcane between $66-81 \%$ among different cultivars with the applications of chemical ripeners. HI is a useful parameter to assess the suitability of different sugarcane cultivars for various products in the industry that can vary under the influence of different chemicals and stresses (Naidu and Venkataramana, 1989). It was noted that all the quality and sugar yielding attributes including juice extraction and purity, sugar recovery and yield was increased by the applications of different chemical ripeners. Chemical ripeners or herbicides can affect the production of ethylene that can induce early maturity. Lee and Dumas (1982) found the changes in ethylene production in tobacco with the applications of Glyphosate. Glyphosate beneficially increased the sucrose contents in sugarcane (McDonald and Jackson, 2001). Changes in ethylene level directly or indirectly regulate the lifespan of plants. Ethylene is tightly correlated with the biosynthesis of volatile organic compounds to create early ripening (Iqbal et al., 2017).

Karmollachaab et al. (2016) observed a beneficial increase in juice pole and brix value in sugarcane by applying Glyphosate and Ethephon for 40 and 65 days respectively. Most of the studies have shown that chemical ripeners inhibited the growth and enhanced the early cane ripening process in various crops including sugarcane. Ethephon inhibited the growth but it also enhanced the tillering and emergence of ratoon with rapid maturity. Different cultivars of sugarcane respond in different ways to Ethephon when it is used as a ripener (Castro et al., 2001; Silva et al., 2007). By applying sulfometuron-methyl, a reduction in pith process (50 to 60\%) was noted with increased sugar yield (Castro et al., 1996). Studies have claimed that sulfometuron-methyl, as a ripener showed consistent improvement in sugarcane brix, pol and reduced pith process (Caputo et al., 2008). Li et al. (2004) compound ripener in sugarcane had an effect on stick's digestion, development and sugar accumulation. It was noted that sulfometuron-methyl did not affect the sugarcane crop yield $\left(\mathrm{t} \mathrm{ha}^{-1}\right)$ and the agronomic features of the crop but was useful for early ripening (Silva et al., 2007; Leite et al., 2010). Different factors can affect the role of chemical ripeners as Solomon and Li (2004) found that cultivar of sugarcane, functional stage of crop at the time of application of ripeners, application rate of chemical, type or combination of ripener and the conditions of growth prior to or after application affected the response of sugarcane to ethephon and glyphosate. 


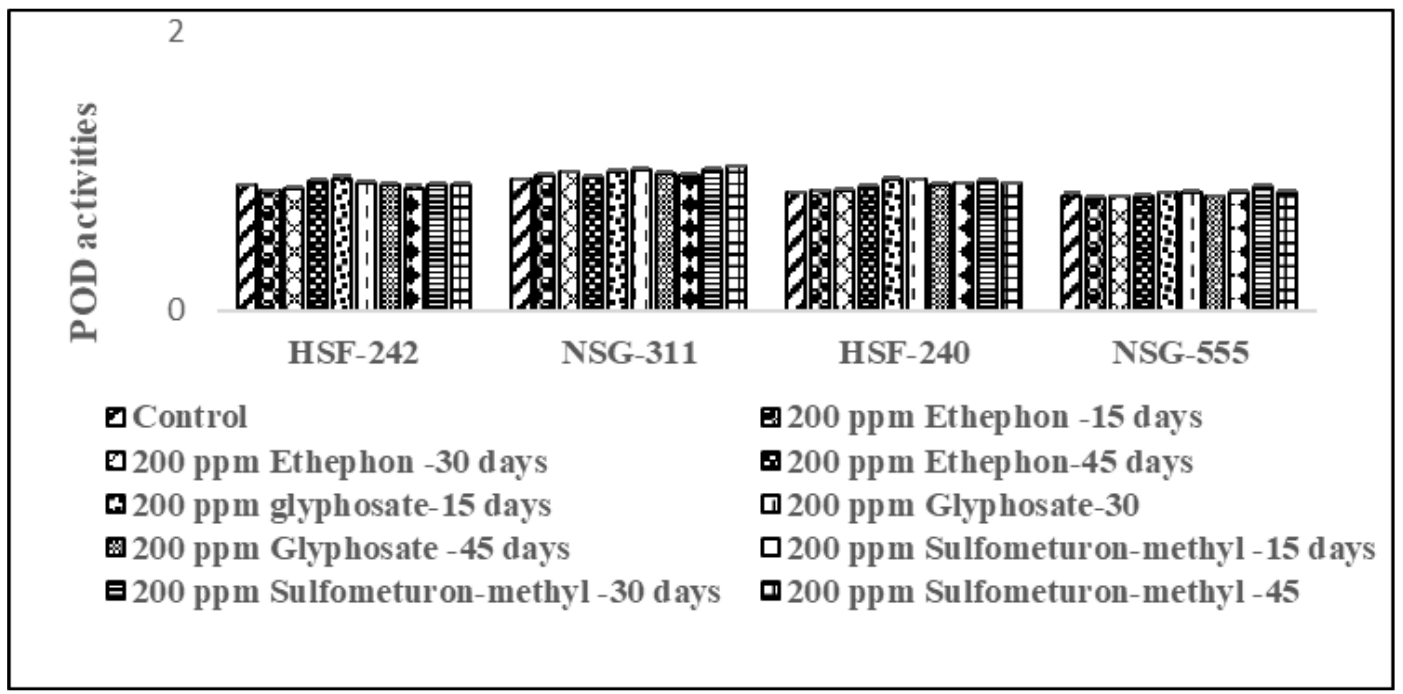

A: Peroxidase (POD)

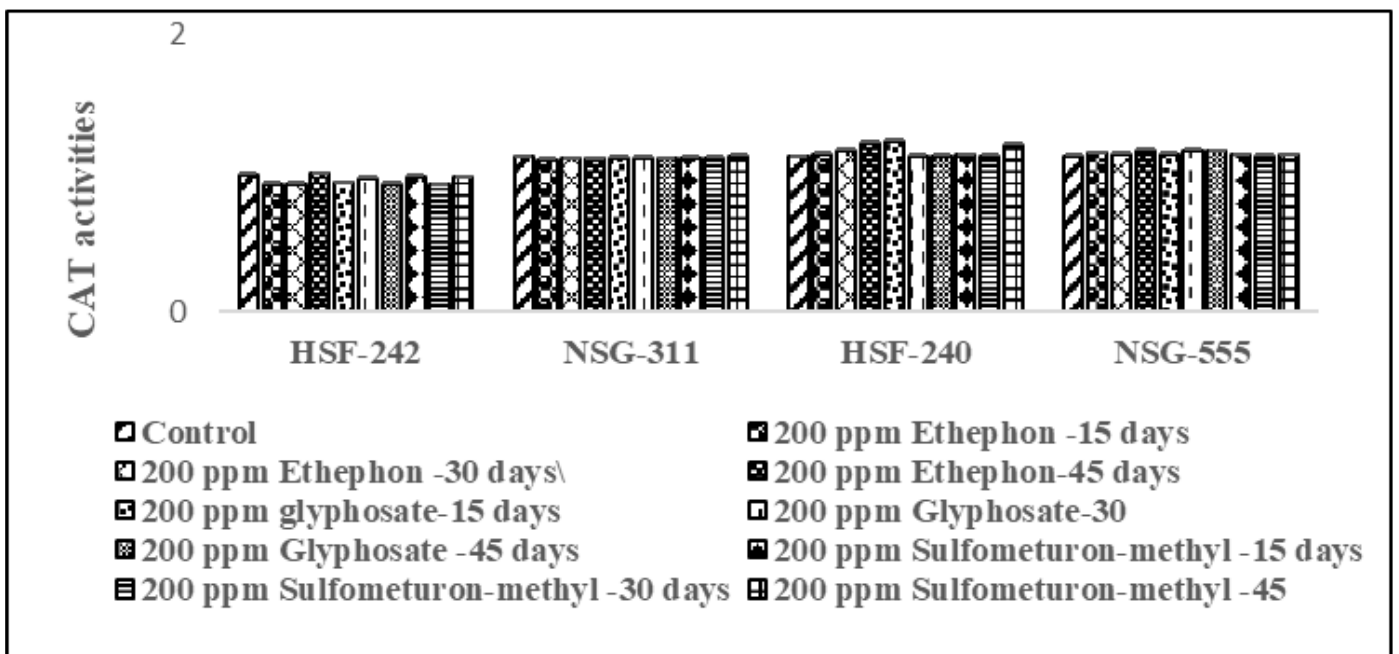

B: Catalases (CAT)

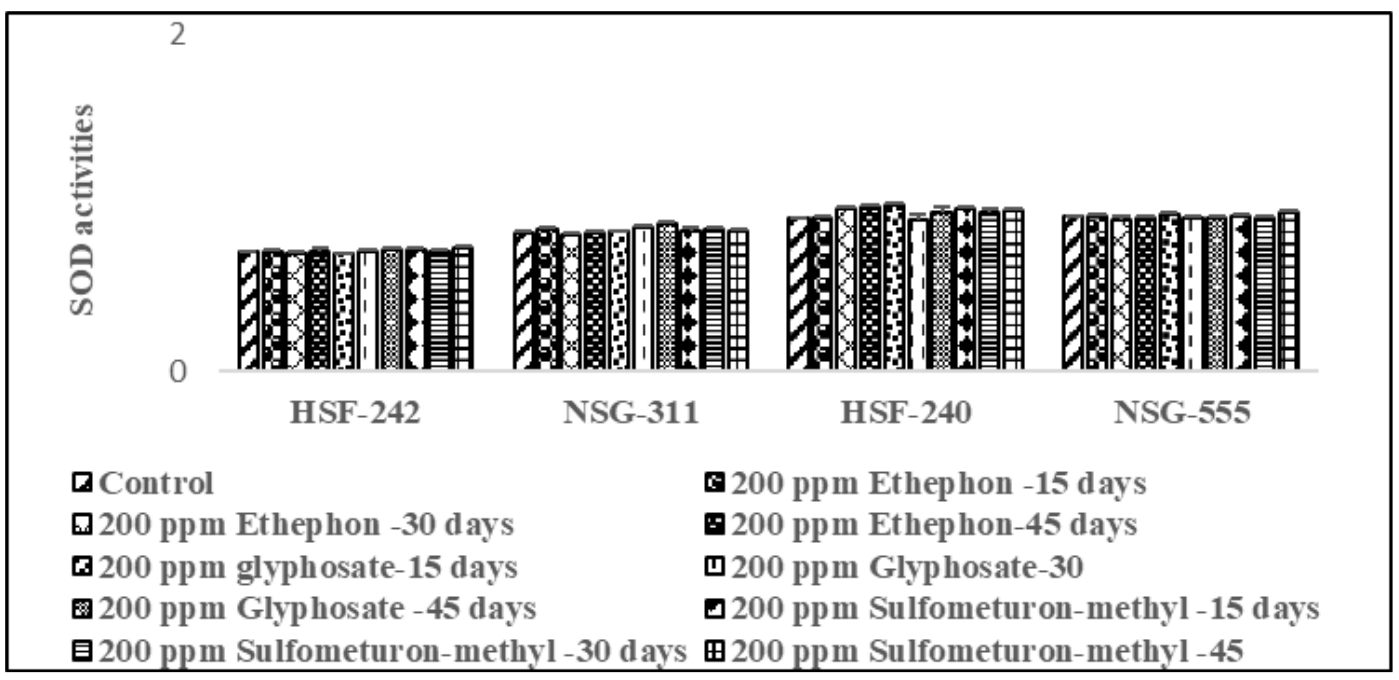

C: Superoxide dismutase (SOD)

Figure 7. Effect of different chemical ripeners on antioxidant activities of sugarcane cultivars in ratooning crop 
During this study the change in antioxidant activities were also noted. Activities of enzymes (CAT, POD and SOD) are a significant index to foretell the plant responses to the changing environments and chemicals (Sen and Mukherji, 2009). In plants, antioxidants activities may act like a defense line for many troublesome conditions and induce early ripening (Lohrmann et al., 2004). Moreira et al. (2020) noted that the Glyphosate $\left(0.15 \mathrm{~L} \mathrm{ha}^{-1}\right)$ and ethephon $\left(0.33 \mathrm{~L} \mathrm{ha}^{-1}\right)$ provided the highest CAT and POD activities without affecting SOD activities of sugarcane. Gill and Tuteja (2010) found that glyphosate increased the CAT activities in sugarcane varieties SP80-1842 and SP80-3280.

In the present studies, it was noted that chemical ripeners had only positive increasing effects for quality and sugar yield for planted crop. It showed non-significant effect on the ratooning abilities, its yield and quality attribute. Ethephon anticipated harvesting stage by minimum 21 days and its residual effect lasted for 60 to 90 days after it had been applied (Caputo et al., 2008). It is well known that chemical ripeners are useful for early cane ripening as well as to enhance the sugar contents and yield in sugarcane but there are some reports showing the negative effect on ratooning abilities. Didier et al. (2017) noted that the applications of glyphosate reduced the sprouting and growth of ratooning crop of sugarcane. Chemical ripener interacted with the production of ethylene for early cane maturity at planted crop but its residual effect was not sustained in ratooning. It was noted that glyphosate negatively affected ratoon and number of stalk. The number and height of cane stalks per unit surface area was reduced (Dalley and Richard-Junior, 2010). Caputo et al. (2008) reported that ethephon showed non-significant effects on sprouting of sugarcane ratoon. Different research studies have reported that non-significant response to sulfometuron-methyl for ratoon of sugarcane. Silva et al. (2007) noted an enhancing effect on the tillering abilities of sugarcane under applications of various chemical ripeners.

\section{Conclusion}

It was concluded that chemical ripeners can be suitable to boost up the sugar yielding characteristics by creating early cane maturity for short term duration before cane harvesting of the crop and it will have no effect on lateral life cycle span and ratooning abilities of the crop.

\section{Recommendations}

It is recommended that chemical ripeners should be utilized to induce early cane maturity in order to achieve higher sugar production from sugarcane.

\section{REFERENCES}

[1] Almendares, A., Usefzadeh, M., Daneshvar, M. (2013): Effect of nitrogen and ethephon on growth parameters, carbohydrate contents and bioethanol production from sweet sorghum. - Sugar Technology 15: 300-304.

[2] Caputo, M. M., Beauclair, E. G. F., Silva, M. A., Piedade, S. M. S. (2008): Response of sugarcane genotypes to the application of maturation inducers. - Bragantia 67: 15-23. 
[3] Castro, P. R., Oliveira, D. A., Panini, E. L. (1996): Action of methyl sulfometuron as a ripener for sugar cane. - Proceedings of $6^{\text {th }}$ Congresso Nacional da Sociedade dos Técnicos Açucareiros de Alcooleiros do Brasil, pp. 363-369.

[4] Castro, P. R., Miyasaki, J. M., Bemardi, M., Marengo, D., Nogueira, M. C. S. (2001): Effect of Ethephon on the maturation and productivity of sugarcane. - Agriculture Magazine 76: 277-290.

[5] Chance, M., Maehly, A. C. (1955): Assay of catalases and peroxidases. - Methods in Enzymology 2: 764-817.

[6] Dalley, C. D., Richard, E. P. (2010): Herbicides as ripeners for sugarcane. - Weed Science 58: 329-333.

[7] De Almeida, S. M., Caputo, M. M. (2012): Ripening and the Use of Ripeners for Better Sugarcane Management. - In: Marin, F. (ed.) Crop Management. Cases and Tools for Higher Yield and Sustainability. InTech Europe, Rijeka.

[8] De Souza, A. P., Gaspar, M., Da Silva, E. A., Ulian, E. C., Waclawovsky, A. J., Nishiyama, J. M. Y., Buckeridge, M. S. (2008): Elevated $\mathrm{CO}_{2}$ increases photosynthesis, biomass and productivity, and modifies gene expression in sugarcane. - Plant, Cell and Environment 31: 1116-1127.

[9] Didier, K. K., Péné, B. C., N'guessan, A. C., Boua, B. M., Ouattara, Y., Zouzou, M. (2017): Effect of glyphosate used as a sugarcane chemical ripener in Côte d'Ivoire. African Journal of Plant Sciences 11(8): 314-350.

[10] El-Hamd, A. S., Bekheet, M. A., Gadalla, A. F. I. (2013): Effect of chemical ripeners on juice quality, yield and yield components of some sugarcane varieties under the conditions of Sohag Governorate. - American-Eurasian Journal of Agricultural and Environmental Sciences 13: 1458-1464.

[11] Gill, S. S., Tuteja, N. (2010): Reactive oxygen species and antioxidant machinery in abiotic stress tolerance in crop plants. - Plant Physiology and Biochemistry 48: 909-930.

[12] Goscinny, S., Hanot, V. (2012): Le glyphosate dans tous ses états. - Institut Scientifique de Santé Publique, Unité Pesticides. Labinfo 7: 12-16.

[13] Iqbal, N., Khan, N. A., Ferrante, A., Trivellini, A., Francini, A., Khan, M. I. R. (2017): Ethylene role in plant growth, development and senescence: interaction with other phytohormones. - Frontiers in Plant Science 8: 475.

[14] Kapur, R., Duttamajumder, S. K., Srivastava, B. L., Madhok, H. L., Kumar, R. (2013): Harvest index and the components of biological yield in sugarcane. - Indian Journal of Genetics 73(4): 386-391.

[15] Karmollachaab, A., Bakhshandeh, A., Telavat, M. M., Moradi, F., Shomeili, M. (2016): Sugarcane yield and technological ripening responses to chemical ripeners. - Sugar Technology 18: 285-291.

[16] Lohrmann, N. L., Logan, B. A., Johnson, A. S. (2004): Seasonal acclimatization of antioxidants and photosynthesis in Chondrus crispus and Mastocarpus stellatus, two cooccurring red algae with differing stress tolerances. - The Biological Bulletin 207: 225232.

[17] Lee, T. T., Dumas, T. (1982): Effect of glyphosate on ethylene production in tobacco callus. - Plant Physiology 72: 855-857.

[18] Leite, G. H. P., Crusciol, C. A. C., Silva, M. D. A., Venturini, F. (2009): Ripeness and technological quality of early harvest sugarcane variety RB855453. - Bragantia 68: 781787.

[19] Leite, G. H. P., Crusciol, C. A. C., Siqueira, G. F. D., Silva, M. D. A. (2010): Technological quality in different sections of the stalk and productivity of sugarcane under the effect of ripeners. - Bragantia 69: 861-870.

[20] Leite, G. H. P., Crusciol, C. A. C., Silva, M. D. A. (2011): Sugarcane development and productivity after application of plant regulators in the middle of the harvest. Agricultural Sciences 32: 129-138. 
[21] Li, Y. R., Zhu, Q. Z., Ye, Y. P., Wang, W. Z., Yang, L. T., Pan, L. Q., Xu, T. (2004): Sugarcane ripening trials with glyphosate-borate complex in commercial plantation of Shansi County, China using micro-light air-craft. - Sustainable Sugarcane and Sugar Production Technology. Proceedings of the International Symposium on Sustainable Sugarcane and Sugar Production Technology, Nanning, Guangxi, China, pp. 560-563.

[22] Ma, J. K., Chikwamba, R., Sparrow, P., Fischer, R., Mahoney, R., Twyman, R. M. (2005): Plant-derived pharmaceuticals-the road forward. - Trends in Plant Sciences 10: 580-585.

[23] McDonald, M., Jackson, P. (2001): The effect of ripeners on the CCS of 47 sugarcane varieties in the Burdekin. - Proceedings Australian Society of Sugarcane Technology 23: 102-108.

[24] Moreira, B. R. A., Viana, P. D., Figueiredo, P. A. M., Lisboa, L. A. M., Magahães, A. C., Ramos, S. B., Viana, C. R., Trindade, V. D., May, A. (2020): Glyphosate plus Carboxylic compounds boost activity of free radical-scavenging enzymes in sugarcane. - Agriculture 10(4): 106-116.

[25] Naidu, M., Venkataramana, S. (1989): Sugar yield and harvest index in water stressed cane varieties. - Sugarcane 6: 5-7.

[26] Neliana, I. R., Sawitri, W. D., Ermawati, N., Handoyo, T., Sugiharto, B. (2019): Development of allergenicity and toxicity assessment methods for evaluating transgenic sugarcane overexpressing sucrose-phosphate synthase. - Agronomy 9: 23.

[27] Sen, S., Mukherji, S. (2000): Season-induced alterations in levels of antioxidants and polygalacturonase activity in tomato (Lycopersicon esculentum Mill.) fruit. - Journal of Environment and Pollution 7: 303-308.

[28] Silva, M. D. A., Gava, G. J. D. C., Caputo, M. M., Pincelli, R. P., Jerônimo, E. M., Cruz, J. C. S. (2007): These cruise control regulators have the potential to be developed and manufactured separately. - Bragantia 66: 545-552.

[29] Solomon, S., Li, Y. R. (2004): Chemical ripening of sugarcane: global progress and recent developments in China. - Sugar Technology 6: 241-249.

[30] Van Heerden, P. D., Mbatha, T. P., Ngxaliwe, S. (2015): Chemical ripening of sugarcane with trinexapac-ethyl Moddus - Mode of action and comparative efficacy. - Field Crops Research 181: 69-75. 